\title{
Design Issues and Considerations for Low-Cost 3-D TSV IC Technology
}

Geert Van der Plas, Member, IEEE, Paresh Limaye, Igor Loi, Abdelkarim Mercha, Herman Oprins, Cristina Torregiani, Steven Thijs, Member, IEEE, Dimitri Linten, Member, IEEE, Michele Stucchi, Guruprasad Katti, Dimitrios Velenis, Vladimir Cherman, Bart Vandevelde, Veerle Simons,

Ingrid De Wolf, Senior Member, IEEE, Riet Labie, Dan Perry, Stephane Bronckers, Nikolaos Minas, Miro Cupac, Wouter Ruythooren, Jan Van Olmen, Alain Phommahaxay, Muriel de Potter de ten Broeck, Ann Opdebeeck, Michal Rakowski, Bart De Wachter, Morin Dehan, Marc Nelis, Rahul Agarwal, Antonio Pullini, Federico Angiolini, Luca Benini, Fellow, IEEE, Wim Dehaene, Senior Member, IEEE, Youssef Travaly, Eric Beyne, and Paul Marchal

\begin{abstract}
In this paper key design issues and considerations of a low-cost 3-D Cu-TSV technology are investigated. The impact of TSV on BEOL interconnect reliability is limited, no failures have been observed. The impact of TSV stress on MOS devices causes Vth shifts, further analysis is required to understand their importance. Thermal hot spots in 3-D chip stacks cause temperature increases three times higher than in 2-D chips, necessitating a careful thermal floorplanning to avoid thermal failures. We have monitored for ESD during 3-D processing and have found no events take place, however careful further monitoring is required. The noise coupling between two tiers in a 3-D chip-stack is $20 \mathrm{~dB}$ lower than in a 2-D SoC, opening opportunities for increased mixed signal system performance. The impact on digital circuit performance of TSVs is accurately modeled with the presented $R C$ model and digital gates can directly drive signals through TSVs at high speed and low power. Experimental results of a 3-D Network-on-Chip implementation demonstrate that the NoC concept can be extended from 2-D SoC to 3-D SoCs at low area $\left(0.018 \mathrm{~mm}^{2}\right)$ and power $(3 \%)$ overhead.
\end{abstract}

Index Terms-3-D, CU TSV, ESD, mechanical stress, network-on-chip, noise coupling, thermal behavior.

Manuscript received April 18, 2010; revised July 16, 2010; accepted July 27 , 2010. Date of publication October 18, 2010; date of current version December 27, 2010. This paper was approved by Guest Editor Alison Burdett.

G. Van der Plas, P. Limaye, A. Mercha, H. Oprins, S. Thijs, D. Linten, M. Stucchi, D. Velenis, V. Cherman, B. Vandevelde, V. Simons, I. De Wolf, R. Labie, N. Minas, M. Cupac, J. Van Olmen, A. Phommahaxay, M. de Potter de ten Broeck, A. Opdebeeck, M. Rakowski, B. De Wachter, M. Dehan, M. Nelis, Y. Travaly, E. Beyne, and P. Marchal are with the Interuniversity Microelectronics Research Center (IMEC), 3001 Leuven, Belgium (e-mail: vdplasg@imec.be).

G. Katti and W. Dehaene are with the Interuniversity Microelectronics Research Center (imec), 3001 Leuven, Belgium, and also with the Katholiek Universiteit Leuven, Department ESAT, 3001 Leuven, Belgium.

C. Torregiani and D. Perry are with Qualcomm (QCT), San Diego, CA 92121 USA.

S. Bronckers is with the EMC Group of Laborelec, 1630 Linkebeek, Belgium. W. Ruythooren is with Photovoltech, Industrie West-Grijpen, 3300 Tienen, Belgium.

R. Agarwal is with the Institute of Microelectronics (IME), Singapore Science Park II, 117685 Singapore.

I. Loi and L. Benini are with the University of Bologna, Bologna 40136 Italy.

A. Pullini and F. Angiolini are with iNoCs, 1007 Lausanne, Switzerland.

Color versions of one or more of the figures in this paper are available online at http://ieeexplore.ieee.org.

Digital Object Identifier 10.1109/JSSC.2010.2074070

\section{INTRODUCTION}

$\mathbf{T}$ HROUGH Silicon Vias (TSVs) are an essential technology towards higher and more heterogeneous system integration. 3-D TSV (through silicon via) technologies promise increased system integration at lower cost and reduced footprint [1], as well as performance improvement such as increased bandwidth and easier reuse by mixing and matching existing silicon. Variants of 3-D technologies have recently been introduced in application areas such as DRAM stacking [2], imagers [3], [4], SSDs (Solid-StateDrives) [5].

In Fig. 1 the different proposed 3-D integration schemes are categorized by their most important feature, via diameter/pitch and via aspect ratio. Three categories are distinguished. The large size 3-D-WLP (Wafer Level Packaging) TSVs have diameters larger than $10 \mu \mathrm{m}$ and serve as bondpad I/O interconnect in systems. They are typically manufactured post-foundry and are compatible with both wafer-to-wafer and die-to-wafer stacking schemes. Because of their rather large size (diameter) small aspect ratios around one or two enable integration in wafers with thickness of $70 \mu \mathrm{m}$ or more, greatly easing wafer and die handling. The medium size 3-D-SIC (3-D Stacked IC) TSVs have diameters between 2 and $10 \mu \mathrm{m}$ and serve as global interconnect. They are manufactured at the foundry and are compatible with wafer-to-wafer and die-to-wafer stacking schemes. An aspect ratio of 5 or higher leads to wafer thickness between $25 \mu \mathrm{m}$ to $70 \mu \mathrm{m}$, making wafer and die handling challenging. The 3-D-SIC TSVs are an emerging technology and are expected to appear in applications in the coming years. The smallest size 3-D-IC TSVs with diameter size of $2 \mu \mathrm{m}$ and smaller target intermediate level interconnect. Even with aspect ratio above 20 they require extremely thinned dies. Their stacking scheme is typically wafer-to-wafer to avoid complex and difficult thin die handling. The 3-D-SIC intermediate level interconnect TSVs are considered risk technology at this time. In this paper, we focus on the emerging $\mathrm{Cu} 3$-D SIC TSV technology, as it is well balanced between cost and application flexibility. It is attractive from cost perspective as it leverages existing CMOS process equipment and it supports die-to-wafer stacking. The latter creates the possibility to stack dies of different sizes, and thus not add unnecessary constraints during system partitioning and floorplanning, which may limit die utilization. 


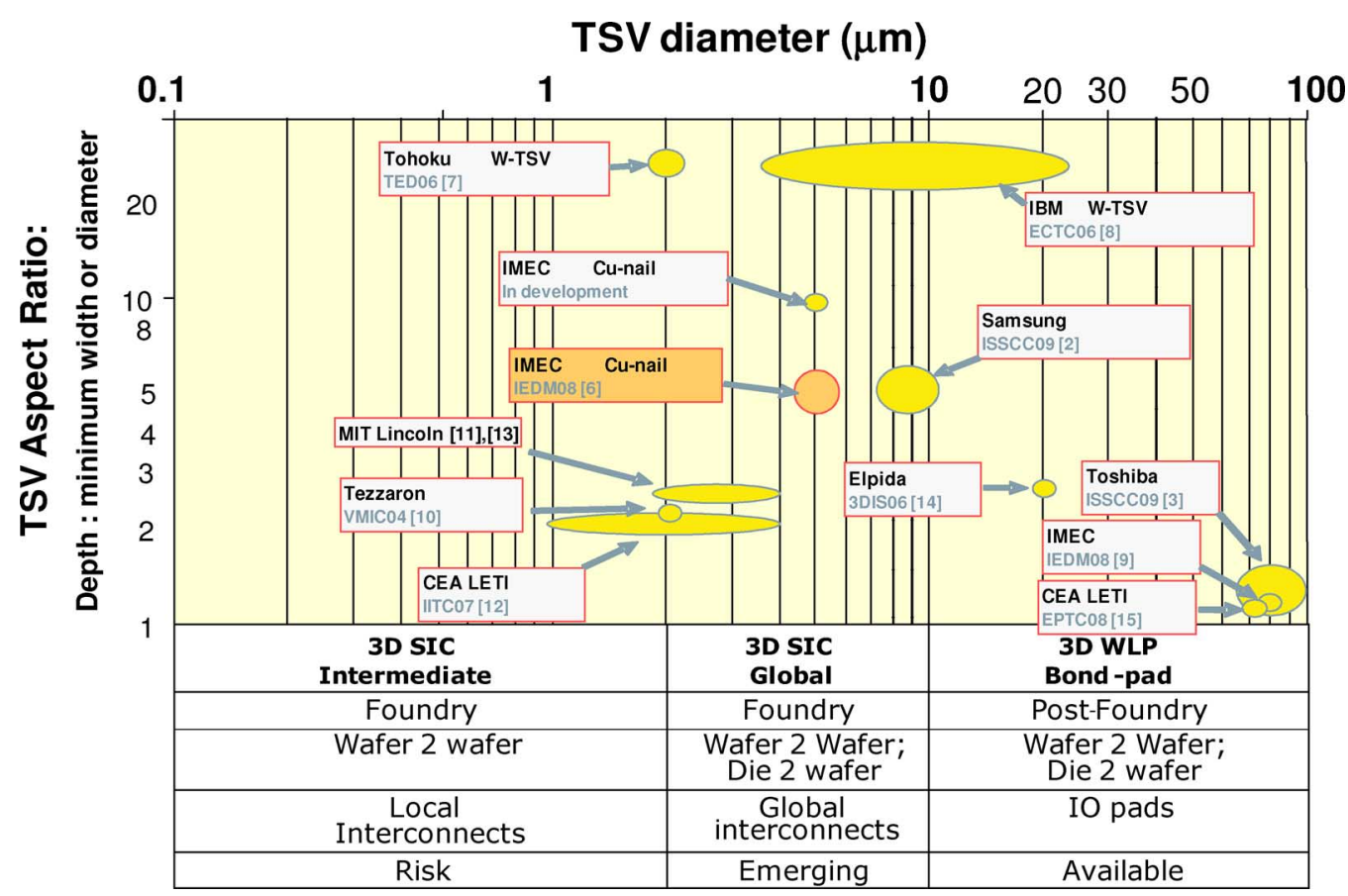

Fig. 1. Overview of 3-D TSV technologies as function of TSV diameter and aspect ratio and classification in 3 categories, 3-D-SIC intermediate and global and 3-D WLP Bondpad with their key attributes.

In this work we investigate in detail the design issues and solutions of a low cost 3-D TSV Stacked-IC technology [16]. The technology we propose offers a $10 \mu \mathrm{m}$ TSV pitch that enables applications such as logic-on-logic, DRAM-on-logic and RF-on-logic. We present experimental data on key issues such as impact of TSV on MOS devices and back-end-of-line (BEOL), reliability, thermal hot spots, ESD, signal integrity and circuit performance. We point out where changes in current design practices are required to realize the low-cost potential of the technology. We also demonstrate a key circuit for the deployment of the Cu TSV technology: a 3-D Network-on-Chip (3-DNoC). The 3-D-NoC will serve as the back-bone communication IP for future 3-D-SoC, as it does today in many 2-D SoCs. We show that the NoC concept can easily be extended to 3-D at low area and power overhead.

This paper is structured as follows. In Section II we introduce the low-cost 3-D Cu TSV technology used in this paper and we review the main characteristics of the Cu TSVs. In Section III the mechanical issues of the 3-D technology are discussed, these are the impact of TSVs on the reliability of the BEOL interconnect and TSV stress impact on MOS devices. In Section IV the thermal behavior of 3-D chip-stacks is discussed and a thermal-aware design approach is proposed to avoid hot spots. In Section V the following electrical issues are reviewed: ESD in 3-D chip-stacks, noise coupling level in 3-D compared to 2-D, and digital signaling through TSV in ring oscillators. In Section VI we present a 3-D-NoC demonstrator circuit that shows the 2-D NoC concept used in SoC is compatible with 3-D technology. In Section VII the main results of the paper are summarized.

\section{LOW COST 3-D CU TSV TECHNOLOGY}

In this section we briefly describe the 3-D technology and test vehicle that was used in the experiments reported in this paper. Next we review the main characteristics of the Cu TSVs, resistance, capacitance, leakage and yield.

\section{A. Technology Description}

The proposed 3-D stacked IC (3-D-SIC) approach leverages existing IC foundry infrastructure to fabricate TSVs after the FEOL processing and prior to BEOL processing [6]. In a 200 $\mathrm{mm} / 130 \mathrm{~nm}$ FEOL CMOS technology with $\mathrm{Cu} / \mathrm{SiO} 2 \mathrm{BEOL}$, TSVs are fabricated with $5 \mu \mathrm{m}$ diameter and a minimum pitch of $10 \mu \mathrm{m}$. After the TSV is etched, an isolation layer is deposited followed by the Cu metallization of the TSV, Fig. 2. The wafers then go through the standard BEOL process. To enable interconnections using TSVs, the wafer is thinned down to $\sim 25 \mu \mathrm{m}$ and next TSVs are exposed to a height of $\sim 700 \mathrm{~nm}$. The thinned wafers are then diced and the resulting dies are stacked face-up on the regular thickness landing wafer with a collective hybrid bonding process in a die-to-wafer approach [17]. This approach reduces the cycle time by the parallel processing of the relatively long $\mathrm{Cu}$ - $\mathrm{Cu}$ thermo-compression step and the die-to-wafer configuration allows the selection of Known Good Die prior to stacking, both reducing overall cost of the 3-D processing.

Using this technology we have built a test vehicle as is shown in Fig. 3. The thinned die is stacked face-up on top of the landing wafer (SEM picture of the stack in Fig. 3). A cross section through a TSV array shows the $25 \mu \mathrm{m}$ high, $5 \mu \mathrm{m}$ diameter $\mathrm{Cu}$ TSVs at a minimum pitch of $10 \mu \mathrm{m}$. The electrical and mechanical $\mathrm{Cu}-\mathrm{Cu}$ bonding is visible at the bottom of the picture, the 

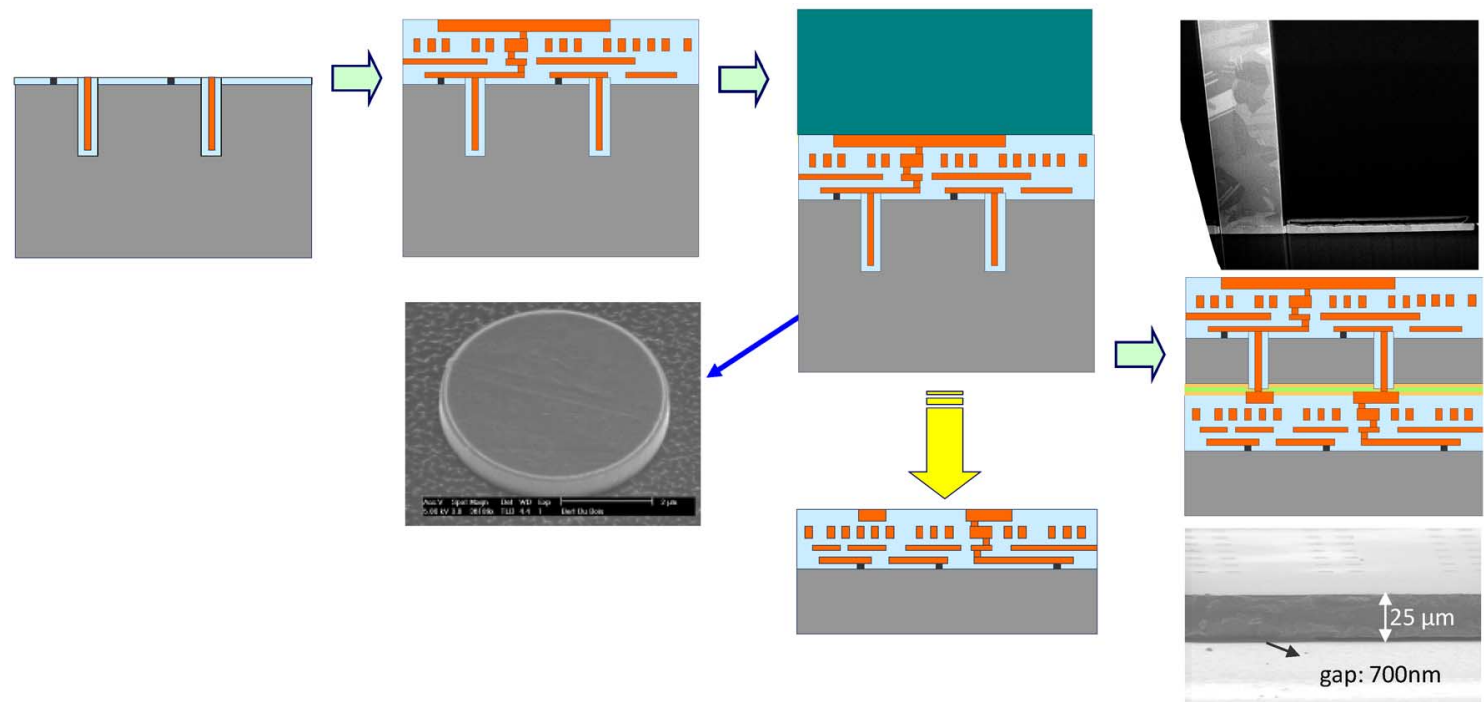

Fig. 2. Process flow of low-cost 3-D Cu TSV technology, Cu TSV are processed after FEOL and before BEOL, next wafers are thinned and dies singulated. Stacking is performed die to wafer with simultaneous $\mathrm{Cu}-\mathrm{Cu}$ thermo-compression to create mechanical and electrical connections simultaneously.
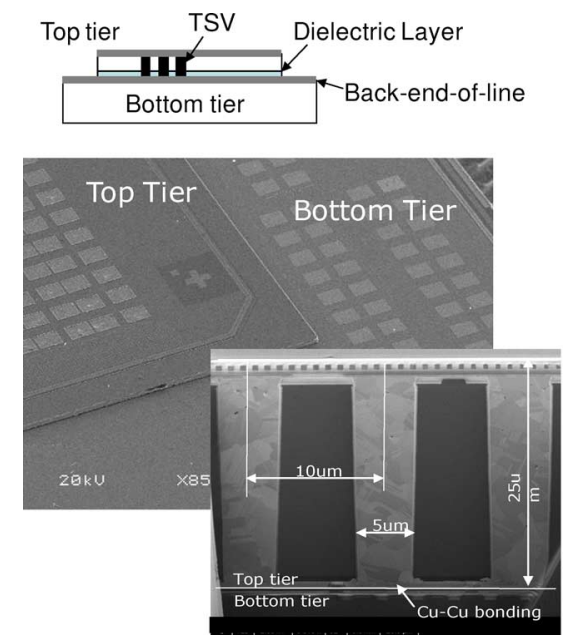

\begin{tabular}{|l|r|}
\hline TSV diameter & $5 \mathrm{um}$ \\
\hline Min. TSV pitch & $10 \mathrm{um}$ \\
\hline Top tier die thickness & $25 \mathrm{um}$ \\
\hline $\begin{array}{l}\text { TSV Isolation layer } \\
\text { thickness }\end{array}$ & $120 \mathrm{n}$ \\
\hline Dielectric layer thickness & $0.7 \mathrm{um}$ \\
\hline
\end{tabular}

Fig. 3. SEM picture of test vehicle with FIB cross section inset (left); summary of dimensions in table (right).

TSV lands on the top metal of the lower tier. The TSV isolation (liner) is $120 \mathrm{~nm}$ thick and the dielectric layer isolating the two tiers is $700 \mathrm{~nm}$ thick. These geometric parameters are summarized in the table in Fig. 3.

\section{B. Electrical TSV Characteristics and Yield}

DC Resistance and low frequency capacitance are fundamental electrical parameters of TSVs. Measure of TSV resistance between the top of the TSV and the landing pad provides information on the quality of the vertical electrical connection established by the bonding of the staked dies. With the proposed TSV dimensions, TSV resistance is expected to be in the order of few tens of milliohms in good TSVs. Therefore, the resistance test structure consists of a single TSV in a 4-point or Kelvin configuration. The Kelvin TSV is placed the four corners and in the center of each stacked die. The measured values show $R_{\text {TSV }} \sim 20 \mathrm{~m} \Omega$; the spread over different die locations and over 17 dies is limited (Fig. 4(a)), thus indicating a good quality of 3-D stacking and bonding.
Dense clusters of TSVs, consisting of arrays of $6 \times 6 \mathrm{TSV}$ where each TSV is measured in a 2 point configuration for assessing the 3-D connectivity only, show an evident pitch dependency of the TSV yield. In particular, TSVs in the array periphery do not provide good electrical connections at $15 \mu \mathrm{m}$ pitch. On the other hand, overall good yield is obtained for $20 \mu \mathrm{m}$ pitch Fig. 4(c).

TSV capacitance is an essential parameter for 3-D circuit design. Since the TSV forms a cylindrical MIS (Metal-Insulator-Semiconductor) capacitor with the substrate, it is expected that this capacitance is non-linear and depends on the biasing of the TSV with respect to the substrate. TSV capacitance values are expected to be in the order of $\sim 100 \mathrm{fF}$. These are too low for the accuracy of a standard LCR meter; therefore, arrays of $32 \mathrm{TSV}$ s connected in parallel are used to measure a larger value of capacitance, which is then averaged over the number of TSVs in the parallel array. De-embedding structures are also characterized to reduce the impact of parasitics on the measurements. C-V plots of TSV capacitance @ 1 MHz (Fig. 4(b)), show a 


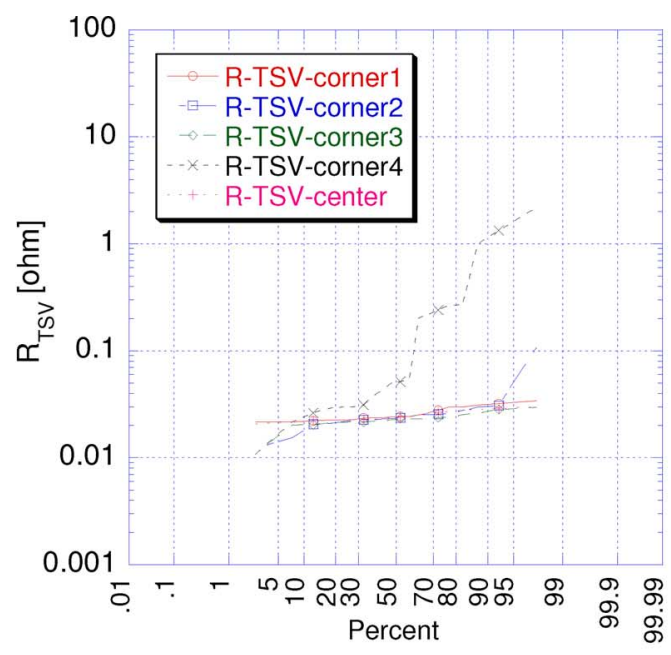

(a)

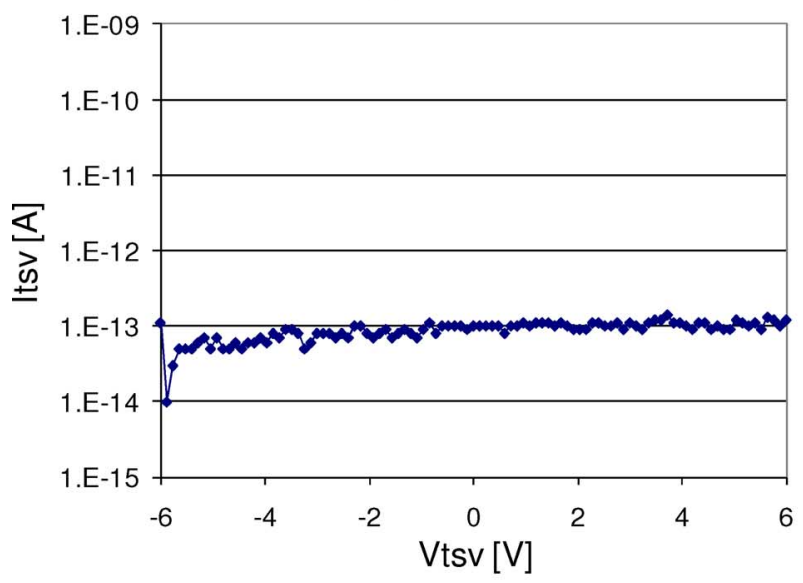

(c)

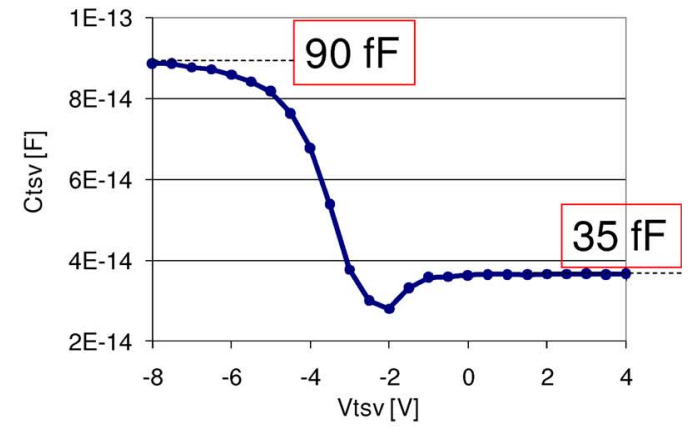

(b)

\begin{tabular}{|l|l|l|l|l|l|}
\hline Yield & \multicolumn{5}{|c|}{ TSV pitch=15um } \\
\hline 0.35 & 0.53 & 0.53 & 0.71 & 0.53 & 0.35 \\
\hline 0.65 & 0.88 & 1.00 & 1.00 & 0.88 & 0.65 \\
\hline 0.71 & 1.00 & 1.00 & 1.00 & 1.00 & 0.76 \\
\hline 0.71 & 1.00 & 1.00 & 1.00 & 1.00 & 0.82 \\
\hline 0.47 & 1.00 & 1.00 & 1.00 & 1.00 & 0.53 \\
\hline 0.29 & 0.47 & 0.65 & 0.35 & 0.24 & 0.24 \\
\hline \multicolumn{7}{|c|}{} \\
\hline Yield & \multicolumn{7}{|c|}{ TSV pitch=20um } \\
\hline 0.76 & 0.88 & 0.88 & 0.82 & 0.94 & 0.76 \\
\hline 0.94 & 0.88 & 1.00 & 1.00 & 0.88 & 0.94 \\
\hline 0.94 & 1.00 & 1.00 & 1.00 & 0.94 & 1.00 \\
\hline 0.94 & 1.00 & 1.00 & 1.00 & 1.00 & 1.00 \\
\hline 0.82 & 1.00 & 1.00 & 1.00 & 1.00 & 1.00 \\
\hline 0.65 & 0.65 & 0.76 & 0.82 & 0.82 & 0.71 \\
\hline
\end{tabular}

(d)

Fig. 4. Summary of TSV characteristics: (a) cumulative distribution of TSV resistance for different locations on the die, (b) TSV capacitance (C-V) as function of bias voltage shows $90 \mathrm{fF}$ in accumulation and $35 \mathrm{fF}$ in depletion mode, (c) TSV leakage as function of bias voltage is well below $1 \mathrm{pA}$ and (d) TSV yield (1.00 is $100 \%$ yield) as function of location of TSV in a $6 \times 6 \mathrm{TSV}$ array with varying pitches, TSVs at the edge of the matrix have lower yield than at center especially at $15 \mu \mathrm{m}$ pitch.

$\mathrm{C}_{\mathrm{TSV}} \sim 37 \mathrm{fF}$ in depletion and a $\mathrm{C}_{\mathrm{TSV}} \sim 92 \mathrm{fF}$ in accumulation mode. TSV leakage, measured in the same array used to measure capacitance, is below $1 \mathrm{pA}$ in depletion and accumulation modes (Fig. 4(d)).

Both $\mathrm{R}_{\mathrm{TSV}}$ and $\mathrm{C}_{\mathrm{TSV}}$ match well with Raphael ${ }^{\mathrm{TM}}$ and Sdevice $^{\mathrm{TM}}$ simulations, respectively, performed by using expected TSV dimensions after processing, with the same methodology proposed in [18].

\section{Mechanical IsSUES AND CONSIDERATIONS IN 3-D TSV TECHNOLOGY}

Due to the difference in thermal expansion coefficient of $\mathrm{Cu}$ and $\mathrm{Si}$, the TSV induces stress on its surroundings [19], potentially leading to reliability problems. To detect reliability problems, back-end-of-line structures such as vias and serpentine wires have been added next to and on top of TSVs. After stacking the test vehicle these structures were characterized and showed no failures. Next, these test structures have been subjected to thermal cycling. After 1000 cycles of $30 \mathrm{~min}$ between $-55^{\circ}$ and $125^{\circ} \mathrm{C}$ no failures have been observed on
17 samples. This is a first level of reliability testing of 3-D TSV technology, further reliability tests are needed to confirm these findings, as well as package level reliability tests to assess lifetimes of systems.

The stress generated by TSV potentially impacts the active device electrical performance, to avoid this proximity effect devices can be spaced away from TSV (keep-out-zone, KoZ) at the expense of increased overall area and cost. The TVS proximity influence on active devices has been investigated for a wide range of physical gate lengths. We observed that the transistors figures of merits like the current factor, the saturation voltage and the drive current are slightly affected by the presence of a TSV in the vicinity of the transistors (Fig. 5). The cumulative probability plot of saturation threshold voltage for a short $(0.13 \mu \mathrm{m})$ and large $(1 \mu \mathrm{m})$ physical gate length of n-type device measured on thinned $(25 \mu \mathrm{m})$ and stacked dies. The threshold voltage shows a slight increase for the longer devices (Fig. 5(b)). This variation has also been measured for the current factor, drive current of n-type devices and also for p-type devices. Further modeling and characterization of the stress impact on MOS 


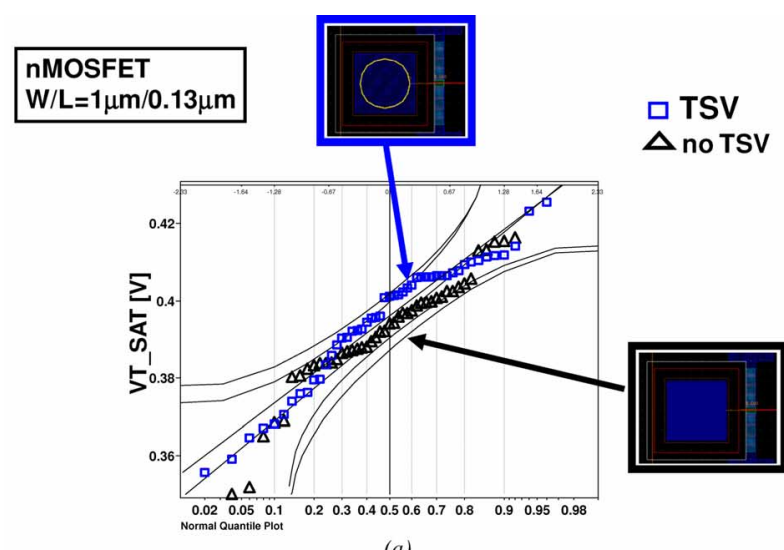

(a)

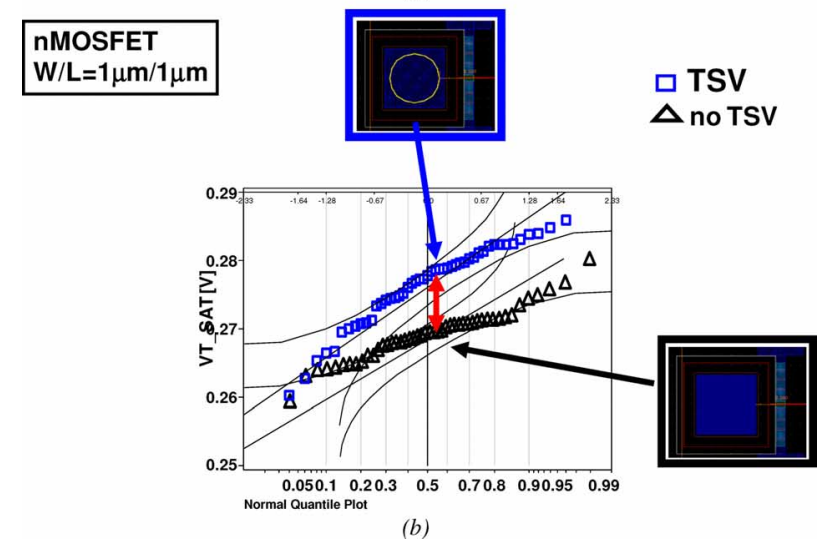

Fig. 5. Impact of TSV proximity on short channel and long channel devices in a $130 \mathrm{~nm}$ technology: no Vth shift is visible on $130 \mathrm{~nm}$ length device (a), about $10 \mathrm{mV}$ th shift is observed on $1 \mu \mathrm{m}$ length device (b).

devices is needed to gain a better insight and asses the severity of this issue. To avoid large keep out areas (areas in which no devices are placed) and increase cost of the use of 3-D TSV technology, models and tools to design for TSV impact on devices are recommended [20].

\section{THERmal IsSUES AND CONSIDERATIONS IN 3-D TSV TECHNOLOGY}

Unless the power dissipation is carefully managed across the tiers in a 3-D stack, hot spots may occur. The reduced thermal spreading in the thinned dies and the poorly thermally conductive adhesives used for the vertical integration, lead to high thermal resistances. The same power dissipation in a stacked die package will lead to higher temperatures and a more pronounced temperature spreading compared to a single die package. To study the thermal impact of hot spot size and power density on 3-D stack design, thermal finite element simulations were performed. Two simulation setups have been used. The fine grain simulation of [21] takes into account the complete BEOL and layout structure whereas in the FEM simulation of [22] simplified models are using volume-averaged material properties. These finite element simulations have been calibrated with a test structure that consists of heaters integrated with thermal sensors (diodes). Heaters with a size of $50 \times 50 \mu \mathrm{m}^{2}$ and $100 \times 100$ $\mu \mathrm{m}^{2}$ are located in the metal 2 layer of the BEOL in the top tier of the 3-D chip-stack, as well as in a 2-D reference die. Both

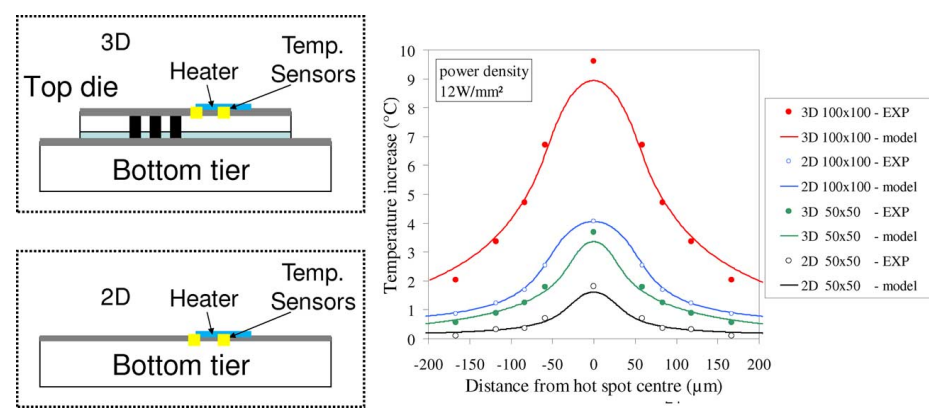

Fig. 6. Temperature increase on the top die in a 3-D chip-stack caused by a $100 \times 100 \mu \mathrm{m}$ hot spot is approximately three times higher (red curve) than the temperature increase in a 2-D SoC chip (blue curve).

in the top and the bottom die of the stack, a set of five diodes at different distances from the hot spot centre are added are integrated below the heater. This configuration of diodes allows capturing the local temperature peak due to the hot spot power dissipation. The simulation results and experimental validation [23] (Fig. 6) indicate that power dissipation in a 3-D stacked structure approximately has a $\sim 3$ times higher maximum temperature increase compared to the 2-D reference case, requiring thermal-aware floor-planning to avoid thermal problems in the stack.

To implement the thermal-aware floor-planning in 3-D stacks, a thermal compact model has been developed [24]. With this model, the temperature distribution is calculated in each die, using the power maps of the heat generation in each tier as input. This compact model allows studying the thermal interaction of heat sources in the 3-D stack, both on the same die as well as on other levels of the stack. Furthermore, the compact model allows thermal optimization of the placement of the heat sources as a function of the geometrical and material properties of the interface and interconnects structures. Fig. 7 shows the graphical interface of this thermal compact model.

\section{ELECTRICAL IsSUES AND DESIGN CONSIDERATIONS IN 3-D TSV TECHNOLOGY}

\section{A. $E S D$}

During the die to wafer stacking process, the top die can discharge through the TSV's into the bottom wafer, resulting in a Charged Device Model (CDM)-like event. The potential need to protect each TSV for ESD may increase the footprint of 3-D connections and hence increase cost of using 3-D technology. Experimental results in the presented technology indicate that no ESD protection is needed and that standard ESD safe-guarding during 3-D process steps is all that is required. Unprotected transistor gates were chosen as monitor since they are most sensitive to ESD events in advanced CMOS technologies. These are connected to TSV's in various connection schemes (Fig. 8). Statistical DC measurements of the leakage current of all ESD monitor variations were collected across the full wafer with stacked dies. The gate-leakage is observed to monitor ESD events, no increase is found (Fig. 8). In total 420 devices were measured over 2 lots and $2.6 \%$ abnormalities 


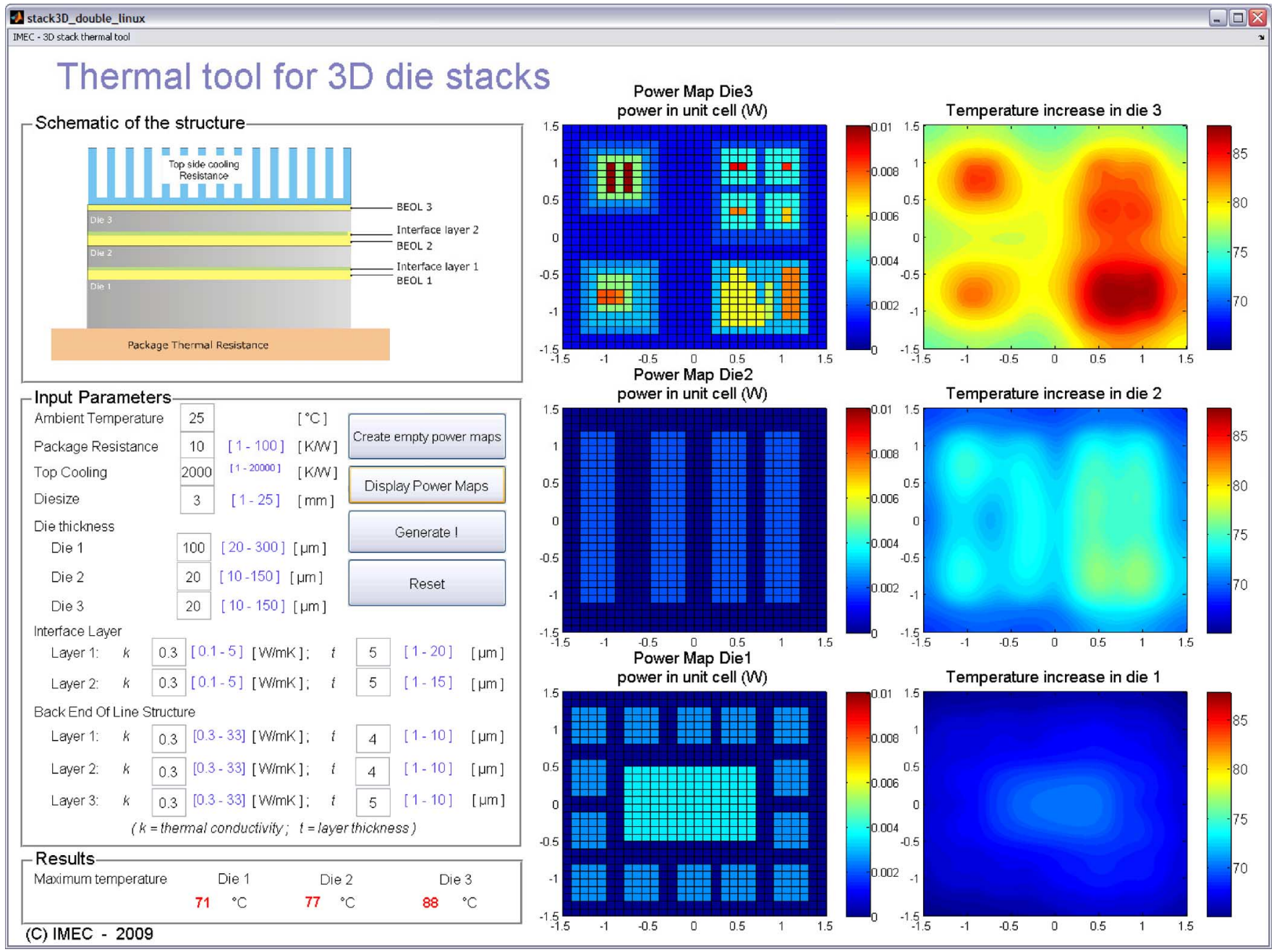

Fig. 7. Graphical interface of the thermal compact model for 3-D stacked structures.

GMON_TOP:

ESD Monitor Reference (Top tier)

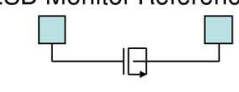

GMON_TOP_TSV:

ESD Monitor 3D+TSV (Top tier)

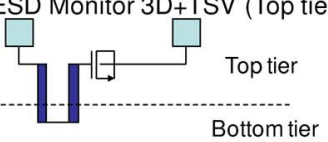

GMON BOT:

ESD Monitor 3D+TSV (Bottom tier)
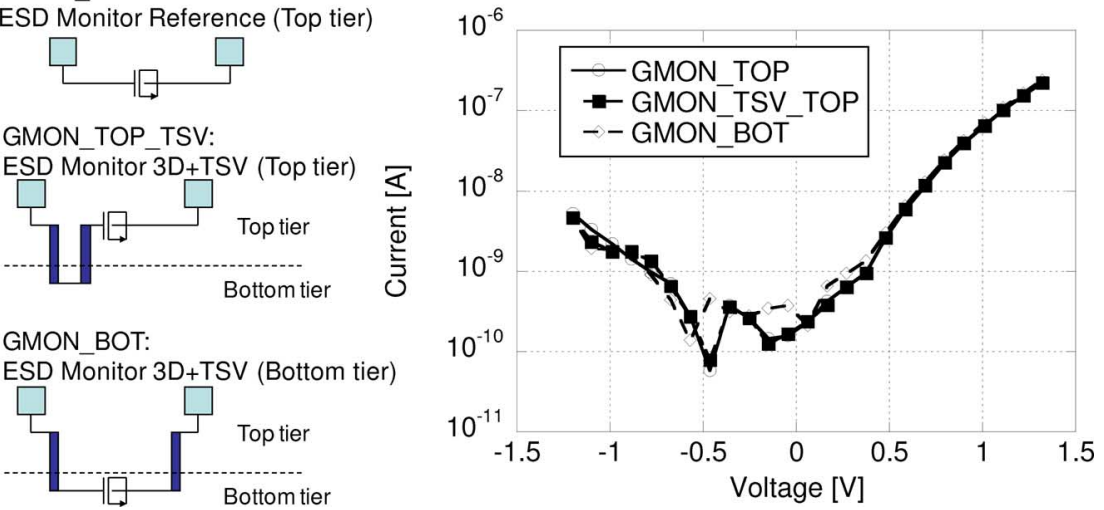

Fig. 8. The leakage currents of ESD monitors (GMON_TOP reference, GMON_TOP_TSV and GMON_BOT) have been measured after stacking: monitors connected through TSVs see no failures (gate shorts) and no significant change versus reference structure GMON_TOP (without TSV).

were detected. These abnormalities resulted consistently in a leakage decrease which excludes gate oxide damage by ESD as a root cause. However, continuous monitoring of future process lots needs to be performed consistently in order to detect possible future ESD occurrences when 3-D technology evolves.

\section{B. Noise Coupling}

Experimental results indicate that substrate noise isolation between stacked tiers is $20 \mathrm{~dB}$ superior compared to 2-D, creating significant opportunities for mixed-signal and RF applications. A $60 \mathrm{GHz}$ voltage controlled oscillator (VCO) circuit has been implemented both in 2-D and on the top tier in a 3-D stack 


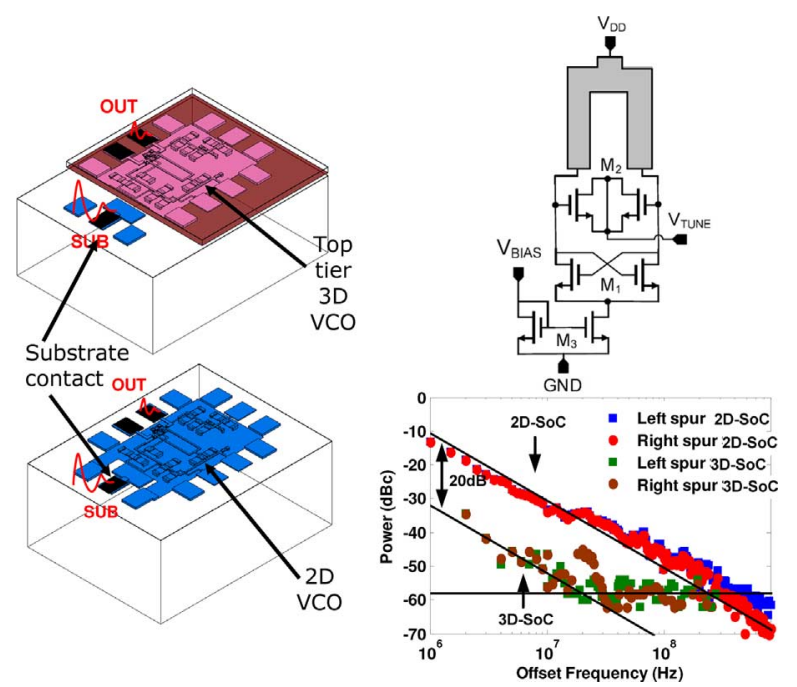

Fig. 9. 2-D SoC and 3-D chip-stack mmWave VCO implementation and schematic: $20 \mathrm{~dB}$ reduction of noise coupling in 3-D versus 2-D is observed.

(Fig. 9) to verify the isolation of 3-D versus 2-D. The VCO performance (center frequency, phase noise) is unaffected by the stacking operation. Both on the 2-D chip and the bottom tier of the 3-D stack a controlled "substrate noise" signal is injected to measure noise coupling as in [25]. This substrate noise signal emulates the switching activity of a digital circuit. The emulating waveform is a sine-wave with a frequency that is varied from $1 \mathrm{MHz}$ to $1 \mathrm{GHz}$, which in practice extracts the coupling in the frequency domain. Due to coupling into the VCO the noise signal appears as unwanted spurs at the output of the VCO at an offset equal to its frequency. The level of the spur power is a measure for the noise coupling, or in other words a lower spur power shows that the isolation is better. The 3-D version exhibits a $20 \mathrm{~dB}$ lower level of spur power than the 2-D SoC variant throughout the frequency range of noise from $1 \mathrm{MHz}$ to $30 \mathrm{MHz}$ beyond which the 3-D spur level drops below noise. This indicates isolation is $20 \mathrm{~dB}$ better in the lower frequency range up to $30 \mathrm{MHz}$ and noise of 2-D-SoC variant exceeds noise in 3-D up to $300 \mathrm{MHz}$ (Fig. 9). This improved isolation capability of 3-D technology opens up opportunities for high performance mixed-signal system design.

\section{Transmitting Digital Signals Through TSVs}

Ring Oscillator (RO) is a standard digital circuit to analyze a particular technology and it is used to verify the feasibility of 3-D circuits and the impact of TSV on digital signaling and circuit operation. 2-D and 3-D RO circuits with varying number of stages and inverter sizes are compared. 21 and 41-stage 3-D ring oscillators with $1 \mathrm{TSV} / \mathrm{stage}$ and without TSVs are implemented as shown in Fig. 10. All RO configurations have their output connected to 8 stage frequency divider (divide by 256) to enable reliable frequency measurements $(0.1-200 \mathrm{MHz})$. To predict the performance of a 3-D circuit, calibrated device models for the transistors along with lumped "T" $R C$ model of the TSV [18] with measured $\mathrm{R}_{\mathrm{TSV}}$ and $\mathrm{C}_{\mathrm{TSV}}$ values are used. Simulation results of power-delay characteristics of the functional RO implementation are well in agreement with measurements suggesting that the model with extracted values can also be extended for predicting the performance of more complex 3-D circuits. It can be also seen that because of smaller inverter sizes the delay exhibited by 21 stage 3-D RO is larger when compared to the delay caused by 41 stage 3-D RO. Hence, in real circuit applications, strong driver elements are necessary to limit the delay caused by TSV in 3-D circuits.

\section{3-D NETWORK ON CHIP DEMONSTRATOR CIRCUIT}

The communication architectures of choice in today's state-of-the-art designs are structured and scalable Networks-on-Chip [26], [27]. The extension of the NoC paradigm to 3 -D integrated circuits is very promising, as modularity and scalability are even more critical for future three-dimensional integrated systems [28].

To demonstrate the feasibility of this communication architecture, we designed and manufactured a 16-bit 3-D NoC distributed across two tiers using the iNoCs synthesizable NoC IP and tool chain, with extensions for supporting vertical links (Fig. 11). Each tier consists of a traffic generator, a slave memory, a $3 \times 3$ switch and a JTAG controller. The traffic generators mimic logic IP components and can send/receive data packets at NoC speed to and from the memory on each tier. A JTAG controller is inserted on each tier to support Known-Good-Die testing before stacking. It also supports testing of the 3-D link after stacking. Note that the test pads of the bottom tier are no longer accessible after stacking. Therefore, these are replicated on the top tier. The PAD SELECT MUX connects these replicated pads to the JTAG bottom block if the top die is present.

A 2.5D design approach was followed to layout the 3-D NOC, i.e., each tier was independently designed but for the TSVs of which the position was aligned on both tiers during P\&R. In total 100 TSVs are used to interconnect both tiers: 12 for $2 \times 6$ for VDD/GND, $2 \times(2 \times 16)$ for the 3 -D link, $3 \times 8$ for the test. Both tiers are manufactured in a the $200 \mathrm{~mm} / 130 \mathrm{~nm}$ FEOL CMOS technology with $\mathrm{Cu} / \mathrm{SiO} 2 \mathrm{BEOL}$ and 3-D $\mathrm{Cu}$ TSV as described in Section I.A. The die-to-wafer configuration allows the selection of KGD prior to stacking, reducing cost of the 3-D processing.

The NoC switches in each tier are connected through a TSV link. Each signal line across the 3-D link is implemented with a standard CMOS buffer (BUFBD2) attached to two TSVs (Fig. 12). TSV duplication per signal is used to protect the link against TSV opens, which is the most frequently occurring fault in our process technology (as described in Section II.B). No ESD protection is used on the 3-D link, as our experimental results indicate that sufficient safe-guarding during 3-D process steps is all that is required (Section V.A).

When activating the NoC, we first enable KGD die testing by ensuring that each 3-D input signal on each tier is driven to a logic value. To this end, we have attached a weak pull down diode to each TSV. The leakage current of the diode in inversion ensures that the input signal of the receiving tier (the TSV_SLECT_MUX) is not floating. Second, we have added the TSV_SLECT_MUX to collect statistics on TSV yield. With this MUX each TSV can be individually tested at boot time through 

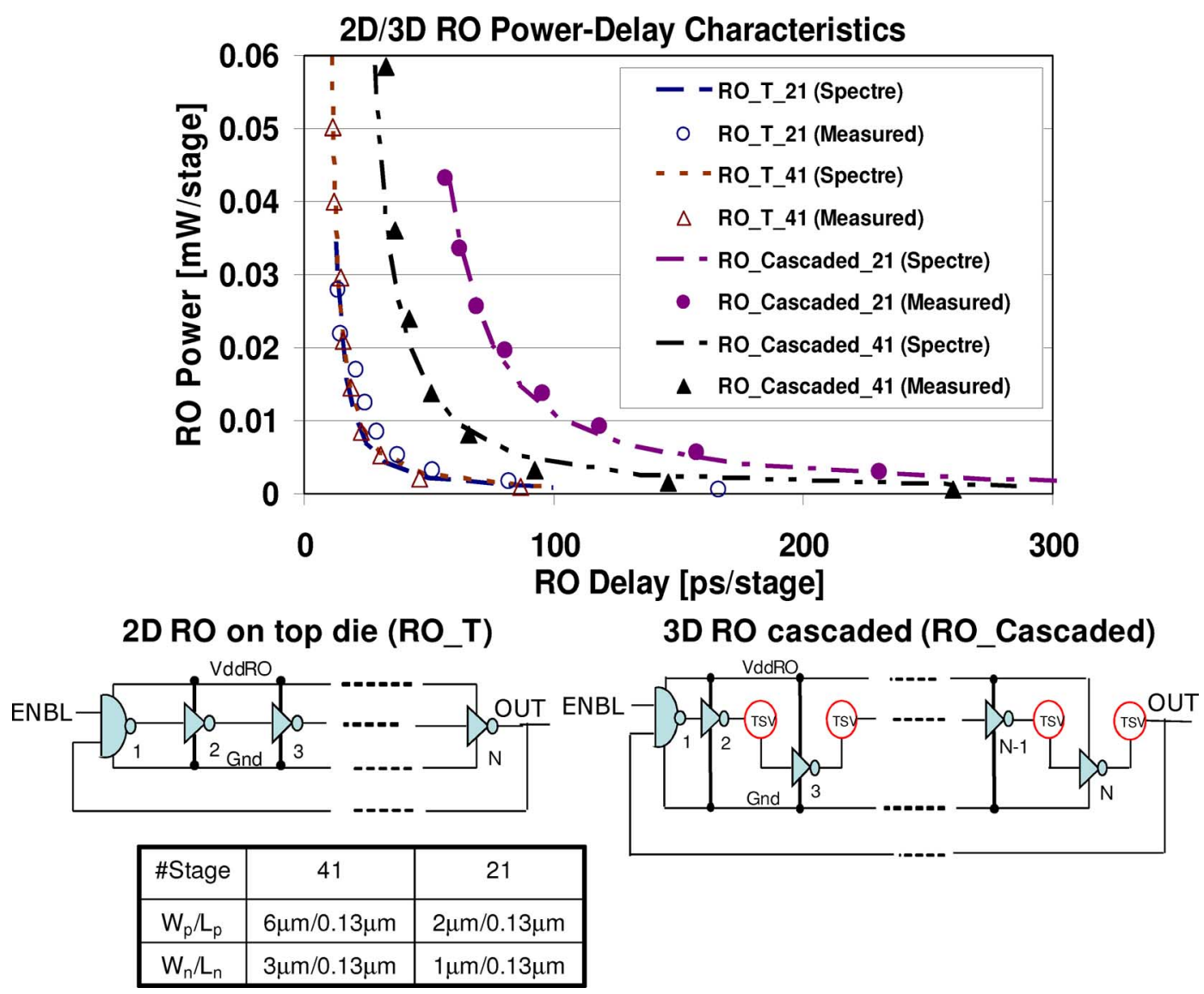

Fig. 10. Ring oscillator schematic (2-D and 3-D), sizing information (21 and 41 stages) and comparison 2-D versus 3-D measurements and simulations.

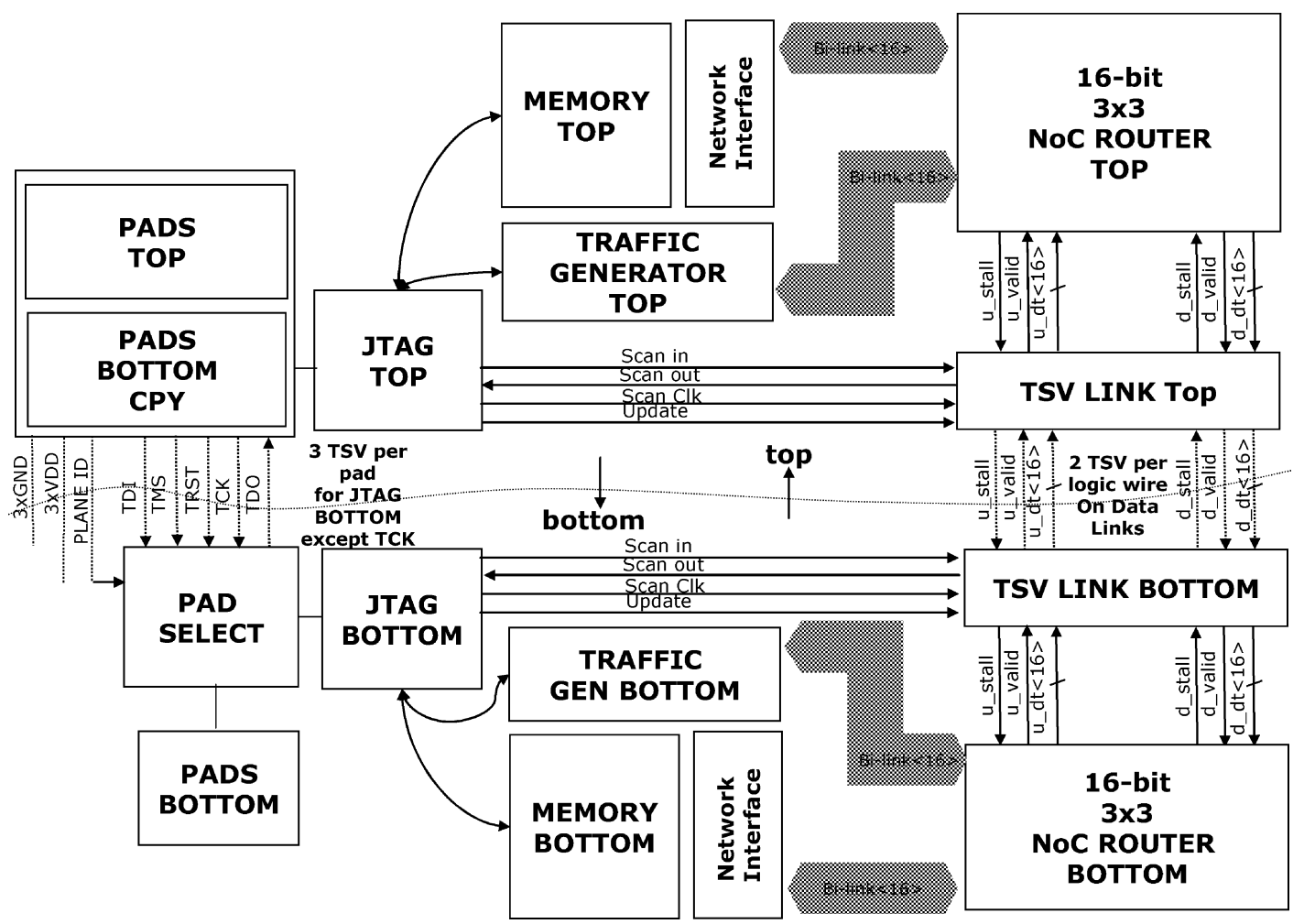

Fig. 11. 3-D NoC schematic, indicated are the circuits embedded in the top tier and the circuits embedded in the bottom tier. The TSVs connecting both circuits are identified and use two or three TSVs per signal to have robustness. 


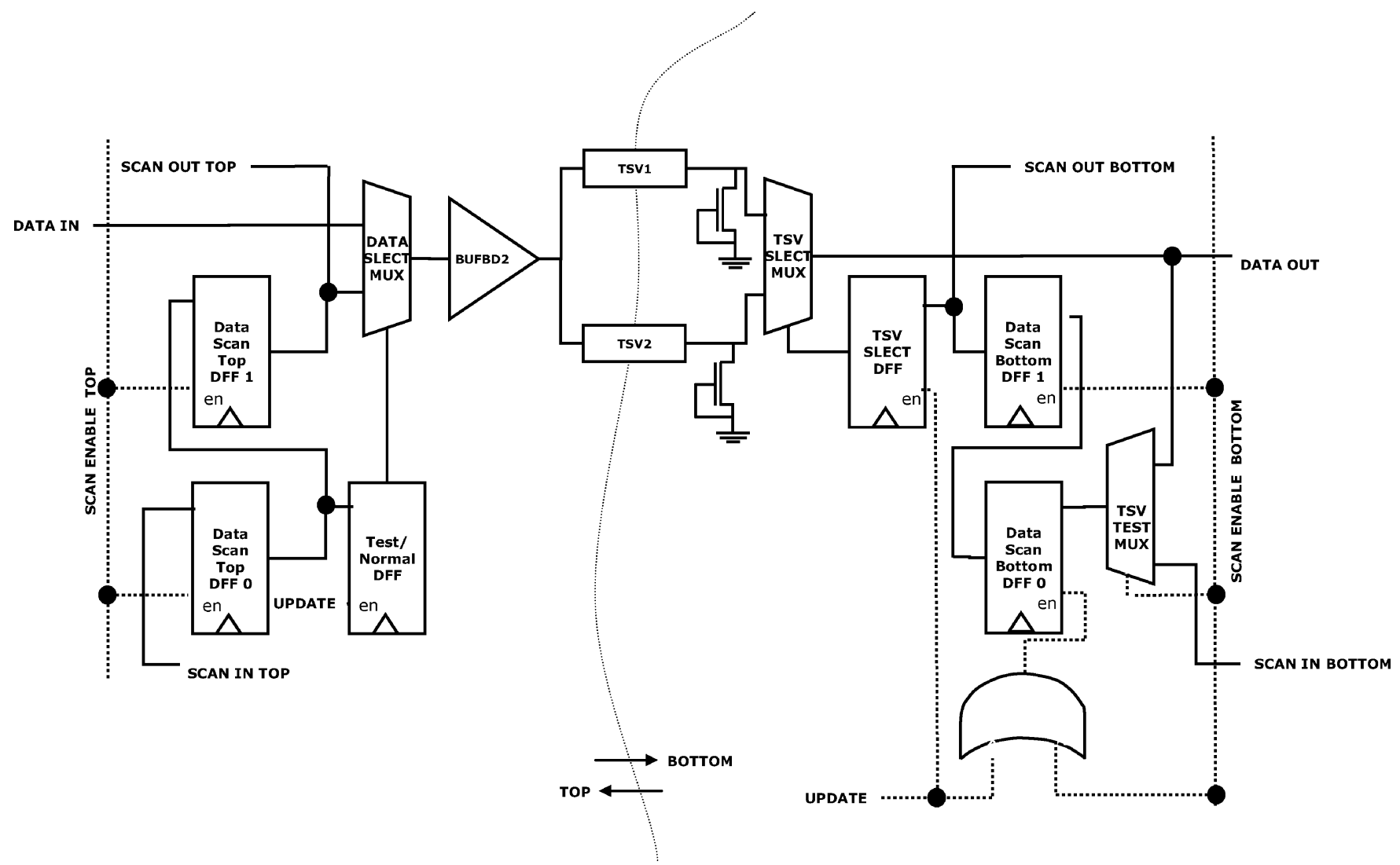

Fig. 12. TSVs for data links are tested using a scan chain and multiplexer. After testing the TSV that functions correctly is selected.

the scan-chain. A test pattern is injected into the scan registers and applied by setting the DATA_SLCT_MUX. After transmitting the data, the receiving register is sampled and scanned in order to detect faulty TSVs. Based on simulation, the redundant TSV and TSV_SLECT_MUX increase the 3-D link delay by $1.6 \times$, and its power consumption by $1.8 \times$ with respect to a single CMOS buffer (BUFBD2) driving a single TSV. In case of a TSV process technology where TSV shorts to bulk frequently occur, the above TSV IO scheme can be adapted by replacing the buffer driving both TSVs with a DEMUX, controlled by additional configuration memories. The DEMUX can then disconnect the failing TSVs from the buffers driving them.

The 3-D NoC operation is depicted in Fig. 13(a). After power on, the design is switched in test mode. First, we program which 32 TSVs out of the total 64 are used for transmitting data on the data link (step 1). Second, we configure what data patterns traffic generators should transmit (step 2). Thereafter, the NoC is switched in operation mode (step 3). In the depicted testcase, short bursts of 32-bits are transmitted in 96-bit network packets. The packets are respectively sent in 3-D across the TSV links from the top(bottom) traffic generator to the bottom(top) memory or in 2-D from the bottom(top) traffic generator to the bottom(top) memory. Finally, the data is scanned out again. The output (tdo) corresponds with the expected output (tdo_exp), confirming a successful operation of the 3-D NoC. Separately, we have tested the 3-D link by transmitted alternating patterns of $0-1 / 1-0$ transitions across the TSV link. So far, we have not found failing TSV on the 3-D NoC link (Fig. 13(b)).
The additional area penalty for the TSVs in the 3-D stack is limited to $0.018 \mathrm{~mm}^{2}$ (Fig. 14). We have placed the TSV interconnect in a regular array of two columns with a pitch of $13 \mu \mathrm{m}$ next to the standard cell core area to optimize yield. In this way we avoided TSV proximity impact as discussed in Section III, at the expense of slightly increased overall area. The power penalty for 3-D data transfers is only 3\% with respect to sending the same continuous stream of 32-bit data bursts in 2-D, between memory and traffic generator spaced at less than $0.5 \mathrm{~mm}$ on the same tier. For 3-D communication at $50 \mathrm{MHz}$ supply $1.39 \mathrm{~mW}$ is consumed from a $1.2 \mathrm{~V}$ compared to $1.34 \mathrm{~mW}$ for 2-D. The delay of the 3-D link is $183 \mathrm{ps}$, enabling high speed data transfers. The 3-D interconnects in the NoC can be designed to operate at a much higher speed. Characterization of ring oscillators driving TSVs shows that data can be transferred across a TSV in less than 150 ps while consuming less than $2 \mathrm{pJ} / \mathrm{bit}$ energy at $1.2 \mathrm{~V}$ (Section V.C). TSVs thus enable transferring of data between dies at a similar speed than intra-die data.

In this section, we have demonstrated a testable 3-D NoC manufactured using a low cost 3-D TSV Stacked-IC technology. This result indicates that modular integration of scalable systems with IP components distributed across multiple dies is feasible.

\section{SUMMARY AND CONCLUSIONS}

In this paper key design issues and considerations of a low-cost 3-D Cu-TSV technology have been investigated. 


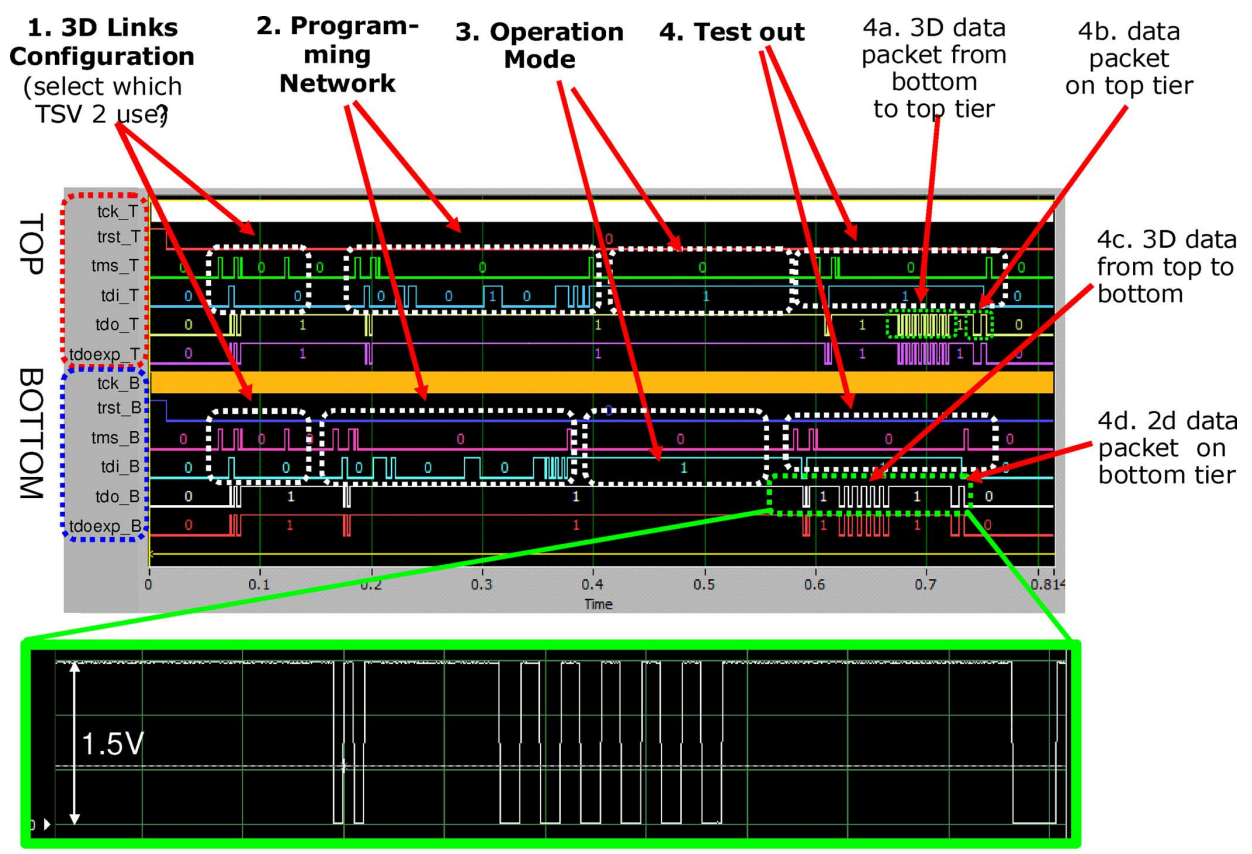

(a)

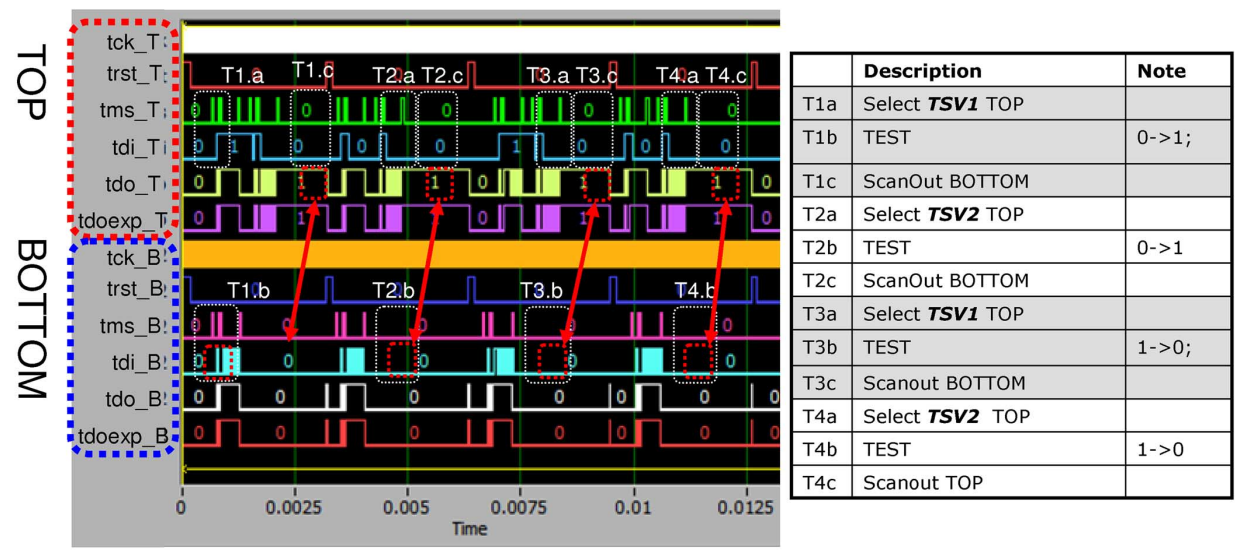

(b)

Fig. 13. Output of scope during wafer-level test; (a) transmission of data burst across TSV links. The expected output (tdo) matches with logic simulation (tdo_exp). Measured maximum performance (25 Mhz@0.4-1.5 V) is limited by wafer-level test setup; (b) JTAG test results of TSVs in d_link (from bottom to top tier); all $38 \mathrm{TSV}$ in this link are functionally working.

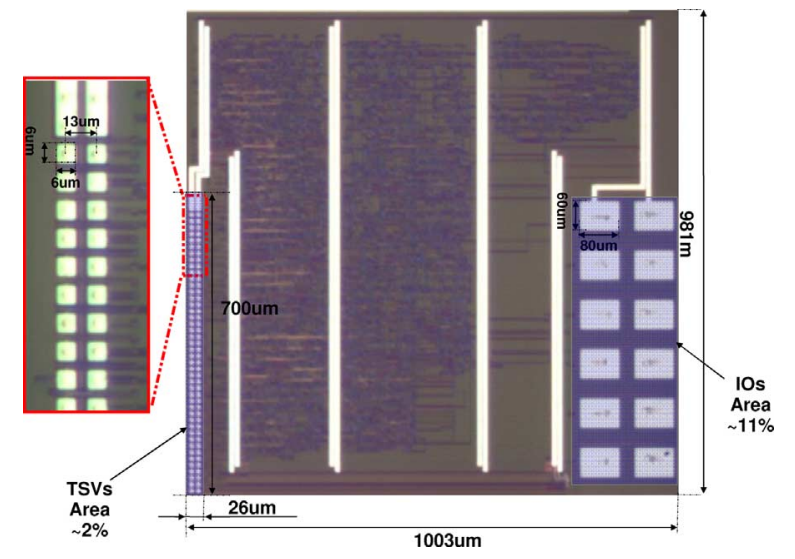

Fig. 14. Microchip photograph of the top tier of the 3-D NoC, indicated are the area consumed by the TSV array $(\sim 2 \%)$, cell NoC core including drivers $(\sim 87 \%)$ and $\mathrm{I} / \mathrm{O}$ area $(\sim 11 \%)$ of the total $1 \times 1 \mathrm{~mm}^{2}$ block.

Thermal cycling while carefully monitoring the integrity of BEOL around TSVs has shown 3-D does not compromise re- liability of BEOL. The impact of TSV stress on MOS devices causes Vth shifts, to account for this during design further analysis and modeling is advised. Thermal hot spots in 3-D chip stacks cause temperature increases three times higher than in 2-D chips, we have proposed thermal floorplanning to take this into account during design. We have found no ESD events during 3-D processing, however careful further monitoring is required. The noise coupling between two tiers in a 3 -D chip-stack is $20 \mathrm{~dB}$ lower than in a 2-D SoC, opening opportunities for increased mixed signal system performance. The impact on digital circuit performance of TSVs is accurately modeled with the proposed $R C$ model and ring oscillators spanning both tiers in the stack show that digital signals can be driven through TSVs at high speed and low power. Experimental results of a 3-D Network-on-Chip implementation demonstrate that the NoC concept can be extended from 2-D to 3-D SoCs at low area $\left(0.018 \mathrm{~mm}^{2}\right)$ and power $(3 \%)$ overhead. 


\section{REFERENCES}

[1] S. Gu et al., "Stackable memory of 3-D chip integration for mobile applications," in Proc. IEDM '08, pp. 1-4.

[2] U. Kang et al., "8 Gb 3-D DDR3 DRAM using through-silicon-via technology," in Proc. ISSCC 09, pp. 130-131, 07.2.

[3] H. Yoshikawa et al., "Chip-scale camera module (CSCM) using through-silicon-via (TSV)," in Proc. ISSCC 09, pp. 476-477, 28.5.

[4] Y. Suntharalingam et al., "A 4-side tileable back-illuminated 3-D-integrated mpixel CMOS image sensor," in Proc. ISSCC 09, 02.1.

[5] K. Ishida et al., "A $1.8 \mathrm{~V} 30 \mathrm{~nJ}$ adaptive program-voltage (20 V) generator for 3-D-integrated NAND flash SSD," in Proc. ISSCC 09, pp. 38-39, 13.5 .

[6] J. Van Olmen et al., "3-D stacked IC demonstration using a through silicon via first approach," in Proc. IEDM, 2008, pp. 303-306.

[7] M. Koyanagi, T. Nakamura, Y. Yamada, H. Kikuchi, T. Fukushima, T. Tanaka, and H. Kurino, "Three-dimensional integration technology based on wafer bonding with vertical buried interconnections," IEEE Trans. Electron Devices, vol. 53, no. 11, pp. 2799-2808, Nov. 2006.

[8] P. S. Andry, C. K. Tsang, E. Sprogis, C. Patel, S. L. Wright, B. C. Webb, L. P. Buchwalter, D. Manzer, R. Horton, R. Polastre, and J. U. Knickerbocker, "A CMOS compatible process for fabricating electrical through vias in silicon," in Proc. 56th Electronic Components Technol. Conf., San Diego, CA, 2006, pp. 831-837.

[9] E. Beyne et al., "Through-silicon via and die stacking technologies for microsystems-integration," in Proc. IEDM, 2008, pp. 1-4.

[10] S. Gupta, M. Hilbert, S. Hong, and R. Patti, "Techniques for producing 3-D ICs with high-density interconnect," in Proc. VLSI Multi-Level Interconnection Conf., 2004, pp. 56-59.

[11] J. Burns, L. McIlrath, C. Keast, C. Lewis, A. Loomis, K. Warner, and P. Wyatt, "Three-dimensional integrated circuits for low-power, highbandwidth systems on a chip," in Proc. Papers IEEE Int. Solid-State Circuits Conf. Tech. Dig., 2001, pp. 268-269.

[12] R. Chatterjeea et al., "Three-dimensional integrated circuits for lowpower, highbandwidth systems on a chip," in Proc. IEEE Int. Interconnect Technol. Conf., 2007, pp. 81-83.

[13] J. A. Burns, B. F. Aull, C. K. Chen, C.-L. Chen, C. L. Keast, J. M. Knecht, V. Suntharalingam, K. Warner, P. W. Wyatt, and D.-R. W. Yost, "A wafer-scale 3-D circuit integration technology," IEEE Trans. Electron Devices, vol. 53, no. 10, pp. 2507-2516, Oct. 2006

[14] T. Mitsuhashi et al., "Development of 3-D-packaging process technology for stacked memory chips," in Proc. Enabling Technologies for 3-D Integration Symp., Boston, MA, Nov. 27-29, 2006, pp. 155-62.

[15] D. Henry et al., "Through silicon vias technology for CMOS image sensors packaging: Presentation of technology and electrical results," in Proc. EPTC 2008, Singapore, Dec. 9-12, 2008, pp. 35-44.

[16] G. Van der Plas et al., "Design issues and solutions for low-cost 3-D TSV IC technology," in Proc. ISSCC 10, pp. 148-149, 07.8.

[17] A. Jourdain et al., "Electrically yielding collective hybrid bonding for 3-D stacking of ICs," in Proc. ECTC, 2009, pp. 11-13.

[18] G. Katti et al., "Electrical modeling and characterization of through silicon via for three-dimensional ICs," IEEE Trans. Electron Devices, vol. 57, no. 1, pp. 256-262, Jan. 2010.

[19] C. Okoro et al., "Extraction of the appropriate material property for realistic modeling of through-silicon-vias using $\mu$-Raman spectroscopy," in Proc. IITC 08, pp. 16-18.

[20] R. Radojcic et al., "Design for manufacturability for fabless manufacturers," IEEE Solid-State Circuits Mag., vol. 1, no. 3, pp. 25-33, Mar. 2009.

[21] H. Oprins et al., "Fine grain thermal modeling of 3-D stacked structures," in Proc. THERMINIC, 2009, pp. 45-49.

[22] C. Torregiani et al., "Thermal analysis of hot spots in advanced 3-Dstacked structures," in Proc. THERMINIC, 2009, pp. 56-60.

[23] H. Oprins et al., "Fine grain thermal modeling and experimental validation of 3-D-ICs," Microelectronics J., Nov. 2009, submitted for publication.

[24] C. Torregiani et al., "A wafer-scale 3-D circuit integration technology," in Proc. EPTC, 2009, pp. 131-136.

[25] C. Soens, G. Van der Plas, P. Wambacq, S. Donnay, and M. Kuijk, "Performance degradation of LC-tank VCOs by impact of digital switching noise in lightly doped substrates," IEEE J. Solid-State Electron., vol. 40, no. 7, pp. 1472-1481, Jul. 2005.
[26] S. Vangal et al., "An 80-Tile 1.28 TFLOPS network-on-chip in $65 \mathrm{~nm}$ CMOS," in Proc. ISSCC, 2007, pp. 98-99.

[27] K. Kim et al., "A 125 GOPS $583 \mathrm{~mW}$ network-on-chip based parallel processor with bio-inspired visual attention engine," in Proc. ISSCC 08, p. 308.

[28] I. Loi et al., "A low-overhead fault tolerance scheme for TSV-based 3-D network on chip links," in Proc. ICCAD 08, pp. 598-602.

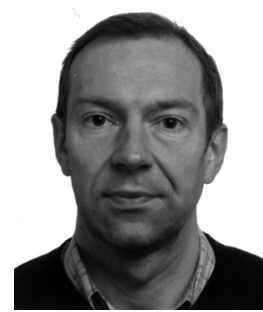

Geert Van der Plas (M'03) received the M.Sc. and $\mathrm{Ph} . \mathrm{D}$. degrees from the Katholieke Universiteit Leuven, Belgium, in 1992 and 2001, respectively.

From 1992 to 2002, he was a Research Assistant with ESAT-MICAS of the Katholieke Universiteit Leuven. He joined imec (SSET), Belgium, in 2003 , where he is a principal scientist. He has been working on energy efficient data converters, low-power scalable radios, power and signal integrity and design technology for 3-D integration. He has authored and co-authored over 100 papers in journals and conference proceedings and serves on the technical program committee of the Symposium on VLSI Circuits.

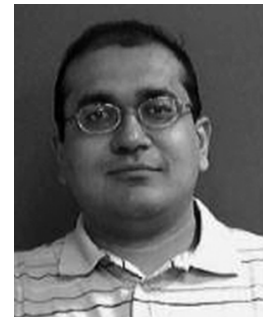

Paresh Limaye received the B.S. degree from University of Pune, India, in 1998, the M.S. degree from the State University of New York at Binghamton in 2000, and the Ph.D. degree from Katholieke Universiteit Leuven, Belgium, all in mechanical engineering.

$\mathrm{He}$ is currently working at the Interuniversity MicroElectronics Center (IMEC), Leuven, Belgium, in the field of 3-D IC stacking and packaging. His main fields of interest and experience include electronics packaging, electronics assembly and manufacturing, finite element analysis, reliability and failure analysis. He has worked in the field of flip chip and SiP packaging at Amkor Technology, Chandler, AZ, and Temex Microsonics, Sophia Antipolis, France.

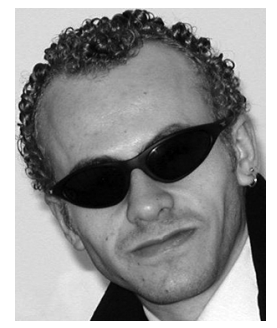

Igor Loi received the B.S. degree in electrical engineering from the University of Cagliari, Cagliari, Italy, in 2005 and the Ph.D. degree in the Department of Electronics and Computer Science, University of Bologna, Italy, in 2010

He is currently holding the position of post-doc in electronic engineering at the University of Bologna. $\mathrm{He}$ is focusing his research on three-dimensional integrated circuit technologies and Networks-on-Chip.

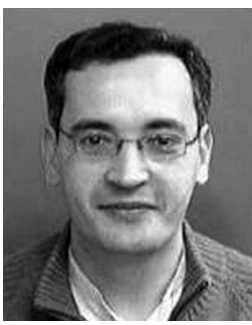

Abdelkarim Mercha received the M.Sc. degree in electrical engineering from the Ecole Nationale Supérieure d'Ingénieurs de Caen (ENSI de Caen), Caen, France, and the Ph.D. degree in microelectronics from the University of Caen, Caen, France, in 1997 and 2000, respectively.

In 2001, he joined the Interuniversity MicroElectronics Center (IMEC), Leuven, Belgium, His interests cover the fields of Analog RF, Logic and Memory devices, 3-D Stacked ICs integration. In these fields, he has (co-)authored over 250 journal and conference papers and two book chapters. He is the editor for the journals Fluctuation and Noise Letters and Active and Passive Electronic Components. 


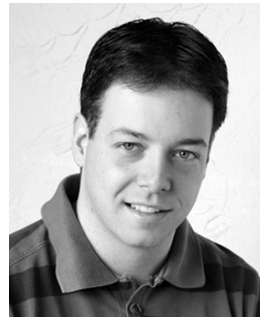

and electrowetting.

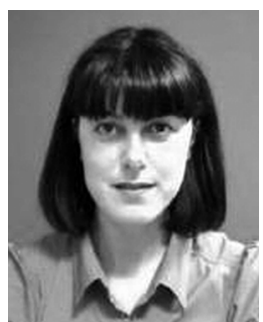

Cristina Torregiani received the M.Sc degree in physics from the University of Bari, Bari, Italy, in 2001, where she also worked from 1999 to 2001 on the development of thin films photocathodes. She received the Ph.D. degree on process-induced stress and strain in planar transistors from the ESAT-INSYS division of the Katholieke Universiteit Leuven, Belgium.

In 2002 she was involved in a research project with IMEC on ultra-shallow-junctions anneal for advanced CMOS applications. In 2007 she joined the Packaging Reliability division of Interuniversity MicroElectronics Center (IMEC), Leuven, Belgium, where she worked on thermal and thermo-mechanical modeling and characterization of packaging for microelectronics. In 2010 she joined Qualcomm, where she overviews the Imec-Qualcomm joint program on 3-D integration as resident in Imec.

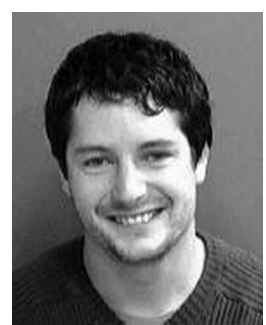

Steven Thijs (M'08) received the M.Sc. and Ph.D. degrees in electrotechnical engineering from the Katholieke Universiteit Leuven (KUL) Belgium, in 2001 and 2009 respectively.

He joined the Silicon Processing and Device Reliability group of Interuniversity MicroElectronics Center (IMEC), Leuven, Belgium, in 2001, where he was involved in ESD protection design, layout, simulation and characterization on CMOS and BiCMOS technologies and in RF circuit design with ESD protection. In 2006, he started working towards a Ph.D. in the field of ESD protection for FinFET devices and for RF CMOS circuits. He has authored and co-authored over 100 publications in the field.

Dr. Thijs received the Best Paper and Best Student Paper Award from the EOS/ESD Symposium in 2004 and 2008 respectively. He served as a member of the Technical Program Committee of the EOS/ESD Symposia 2007-2010 and IRPS 2009. He is a member of IEEE and a peer reviewer for various IEEE journals.

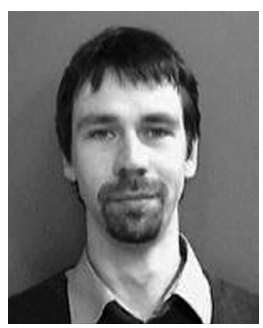

Dimitri Linten (S'01-M'06) received the M.Sc. and $\mathrm{Ph} . \mathrm{D}$. degrees in electrical engineering from the Vrije Universiteit Brussel (VUB), Brussels, Belgium, in 2001 and 2006, respectively.

In 2001, he joined the Wireless Research group of Interuniversity MicroElectronics Center (IMEC), Leuven, Belgium In 2006, he joined the department Fundamental Electricity and Instrumentation (ELEC) at the Vrije Universiteit Brussel and the ESD reliability group at IMEC as a post-doctoral research fellow, supported by the Institute for the Promotion of Innovation through Science and Technology in Flanders (IWT-Vlaanderen). In 2007, he became the scientific coordinator of the ESD research group at IMEC. His research interests are on-wafer ESD testers, ESD-reliable RF circuit design and ESD reliability for sub-32 nm finfet and planar devices, high voltage silicon technologies, Radiation and Python OOP. He authored or co-authored more then 100 publications and patents in these fields.

Dr. Linten received the Best Paper Award from the EOS/ESD Symposium 2004. In 2010, he co-founded an Open Source Initiative on ESD data analysis Software He is serving in the TPC of ESREF, ICICDT and IEW. He is a member of Management Committee of the International ESD Workshop 2011 (IEW).

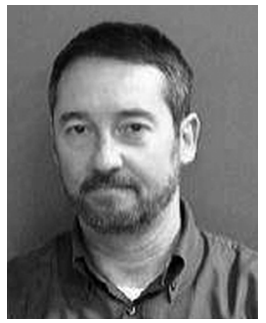

Michele Stucchi received the M.S. degree in electrical engineering from the University of Bari, Italy, in 1988.

From 1989 to 1991 he worked as interconnect and silicide process engineer for DRAM plant of Texas Instruments, Avezzano, Italy, and from 1991 to 1996 as an engineer in failure analysis, FIB analysis and in ESD and latch-up characterization of IC devices in Tecnopolis science park, Bari, Italy. In 1996 he joined Interuniversity MicroElectronics Center (IMEC), Leuven, Belgium, where he managed the Technology-Aware Design research program. He currently works as Senior Research Engineer in characterization, modeling and reliability aspects of 2-D and 3-D interconnects.

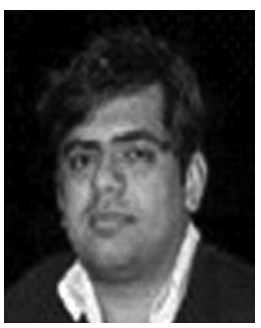

Guruprasad Katti received the B.E. degree in electrical engineering from A.C. Patil College of Engineering, University of Mumbai, Mumbai, India, and the M.S. degree in electrical engineering from the Indian Institute of Technology Madras, Chennai, India, in 1999 and 2002, respectively. He is currently pursuing the Ph.D. degree in Interuniversity MicroElectronics Center (IMEC), Leuven, Belgium, and Katholieke Universiteit Leuven (KUL) on 3-D IC interconnects.

Prior to joining the Ph.D. program he worked with GE-Global Research and National Semiconductor, Bangalore, India.

Mr. Katti is a recipient of the IEEE EDS Ph.D. Student Fellowship Award for the year 2010. His research interests include 3-D ICs, IC interconnects and novel device structures.

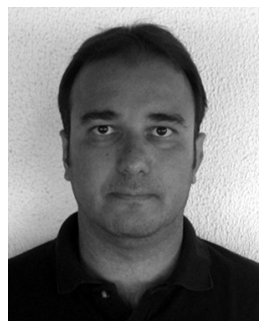

Dimitrios Velenis received the M.Sc. and Ph.D. degrees from the University of Rochester, Rochester, NY, in 2000 and 2003, respectively.

He was an Assistant Professor with the Department of Electrical and Computer Engineering, Illinois Institute of Technology, until Fall 2007 and a Research Associate at the University of Rochester until Spring 2008. Since April 2008 he has been with Interuniversity MicroElectronics Center (IMEC), Leuven, Belgium, working on characterization and cost modeling of 3-D integration processing. He has been the author or coauthor of over 30 papers in journals and conference proceedings.

Dr. Velenis was the recipient of the 2004 Outstanding Dissertation Award from the European Design Automation Association.

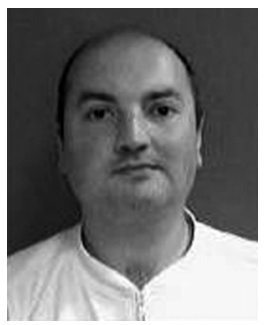

Vladimir Cherman received the M.Sc. and Ph.D. degrees in electronic engineering from Saint Petersburg Electrotechnical University (ETU-LETI), Russia, in 1994 and 1999, respectively.

From 1997 to 2000, he was with Morion Inc., Saint Petersburg, Russia, as an electronics engineer. From 2000 to 2007 he was with Ceramics Laboratory at Swiss Federal Institute of Technology Lausanne (EPFL) where his research was focused on microwave properties of ferroelectric materials. He joined the Reliability and Modeling group of Interuniversity MicroElectronics Center (IMEC), Leuven, Belgium, as a Researcher in 2007, where he is involved in reliability study of MEMS devices and electronic packages. 


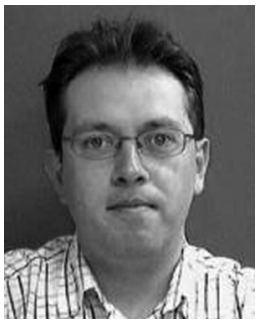

Bart Vandevelde received the M.S. degree in mechanical engineering from the Catholic University of Leuven, Belgium, in June 1994. In March 2002, he received the $\mathrm{Ph} . \mathrm{D}$. degree at Interuniversity MicroElectronics Center (IMEC), Leuven, Belgium, in the field of thermo-mechanical modeling for electronic systems.

Currently, he is team leader for the packaging reliability activities at IMEC and internal project coordinator for several Flemish and European projects. He is co-founder and member of the organization committee for the Eurosime conference.

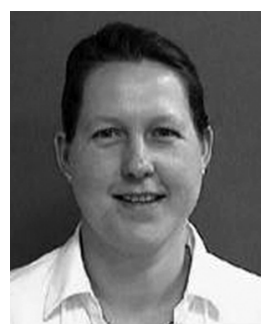

Veerle Simons is currently working in the reliability group (INTPAC/REMO) of the Process Technology unit at Interuniversity MicroElectronics Center (IMEC), Leuven, Belgium.

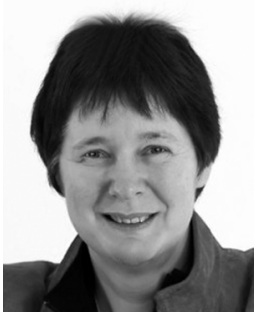

Ingrid De Wolf (SM'07) has been with Interuniversity MicroElectronics Center (IMEC), Leuven, Belgium since 1989.

She performed research on reliability physics of semiconductor devices, with special attention for mechanical stress aspects and failure analysis. Since 1999, she heads the group REMO (Reliability and Modelling), where research is focused on test and reliability of MEMS and IC-interconnect and packaging, including 3-D. She is a part time Professor in the Metals and Applied Materials Engineering Department of the Katholieke Universiteit Leuven, Belgium.

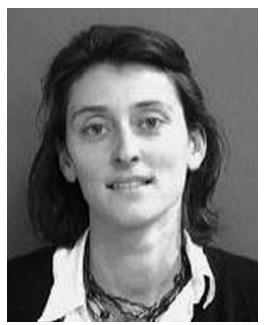

Riet Labie received the M.Sc. and Ph.D. degrees in materials science from the Katholieke Universiteit Leuven, Belgium, in 1999 and 2007, respectively.

She joined Interuniversity MicroElectronics Center (IMEC), Leuven, Belgium, in 1999, where she first worked as Process and Research Engineer in packaging and flip chip interconnections. In 2008, she started working as a reliability engineer for packaging and interconnects, with particular focus on 3-D.

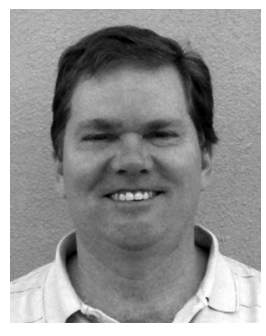

Dan Perry received the B.S.E.E. degree from the University of California at San Diego.

$\mathrm{He}$ is a Staff Engineer at Qualcomm working on 3-D integration issues. He recently completed a two year assignment at Interuniversity MicroElectronics Center (IMEC), Leuven, Belgium. His previous assignments at Qualcomm include evaluation of design-for-manufacturability tools and design enablement. Prior to joining Qualcomm, he held engineering positions with PDF Solutions and Cadence Design Systems.

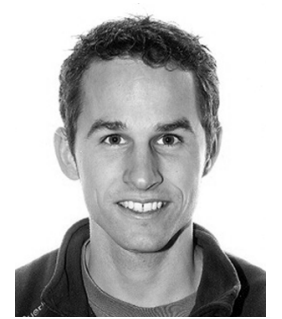

Stephane Bronckers received the M.S. degree in engineering from the Vrije Universiteit Brussel (VUB), Belgium, in 2005, and the Ph.D. degree from Interuniversity MicroElectronics Center (IMEC), Leuven, Belgium.

In 2006 he joined the Fundamental Electricity and Instrumentation (ELEC) Department at the VUB as a research assistant supported by the Institute for the Promotion of Innovation Through Science and Technology in Flanders (IWT-Vlaanderen). He worked at the Wireless Group of IMEC. His research focused on developing a methodology to improve the electromagnetic immunity of analog integrated RF circuits. In 2009, he joined the EMC Group of Laborelec, which is part of the GDF-Suez group.

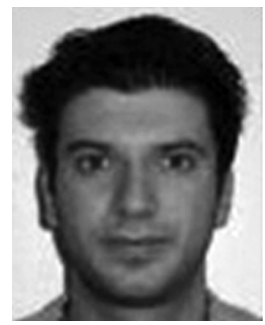

Nikolaos Minas received the M.Sc. and Ph.D. degrees from Newcastle University, U.K.

Since 2008, he has been with imec (SSET), Belgium, where he is working as a researcher in the area of 3-D Stacked Integrated Circuits (3-D-SIC).

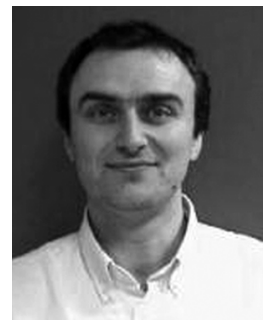

Miro Cupac is currently working in the Digital Components Department (DC) of the Smart Systems and Energy Technology unit at Interuniversity MicroElectronics Center (IMEC), Leuven, Belgium.

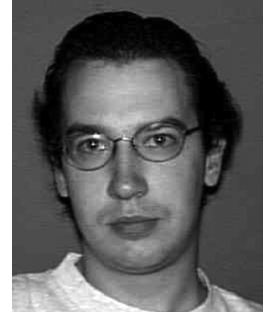

Wouter Ruythooren received the Ph.D. degree in materials engineering from Katholieke Universiteit Leuven, Belgium, for a study on electrodepostion of magnetic materials in 2002.

He then initiated the development of GaN HEMT devices at Interuniversity MicroElectronics Center (IMEC), Leuven, Belgium. In 2005, he took up responsibility for advanced packaging activities, including development of TSV metallisation and metal bonding for 3-D stacking technology. Still at IMEC, in 2009 , he became manager of all electroplating activities. Since July 2010, he has been working in process development for solar cells at Photovoltech, Belgium.

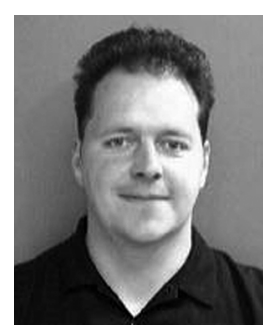

Jan Van Olmen is currently back-end of line Integration Engineer in the packaging group (INTPAC/PAMAHT) of the Process Technology unit at Interuniversity MicroElectronics Center (IMEC), Leuven, Belgium. 


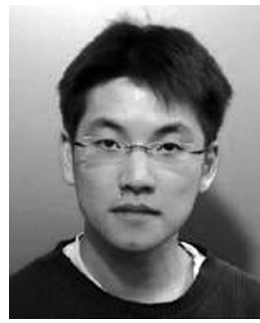

Alain Phommahaxay received the Ph.D. from the ESYCOM-ESIEE, Noisy-le-Grand, France, in 2007.

Since 2007, he has been with Interuniversity MicroElectronics Center (IMEC), Leuven, Belgium, where he has been working in the litho group of the Process Technology unit.

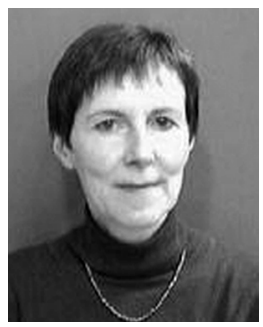

Muriel de Potter de ten Broeck received the M.S. degree in physics in 1978 and the Ph.D. degree in 1984, both from the Katholieke Universiteit Leuven in Leuven, Belgium.

From 1978 to 1984 she was a research assistant at the IKS (Instituut voor Kern-en Stralings Fysika) of the University of Leuven. In 1984 she joined the newly established R\&D laboratory IMEC (Interuniversity Micro-electronics Center) in Leuven, Belgium, as a member of the Compound Semiconductor Processing group. She has been working on ion implantation in GaAs and on the development of advanced GaAs MESFET's (microwave field-effect transistors). In 1995 she moved to the Interconnect Technologies and Silicides department within the Silicon Process Technology Division. Since 2004 she is active, as a Senior Process Engineer, in the baseline process development for CMOS platform within the Process Technology division.

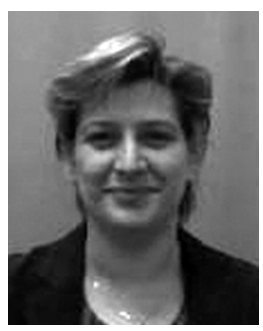

Ann Opdebeeck is currently Process Assistant at Interuniversity MicroElectronics Center (IMEC), Leuven, Belgium.

From 2005 to 2006 she worked as a Customer Assistant at NCI business center.

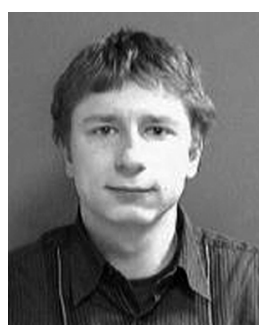

Michal Rakowski received the M.Sc. degree in electronic engineering from the Warsaw University of Technology, Poland, in 2006.

Since 2006, he had been with the Institute of Microelectronics and Optoelectronics (VLSI Engineering and Design Automation Division), at the same university, working as a lecture assistant and pursing his Ph.D. From 2008 he has been with IMEC (Process Quality and Integration), Belgium, where he has been working on an electrical data analyzes and measurements.

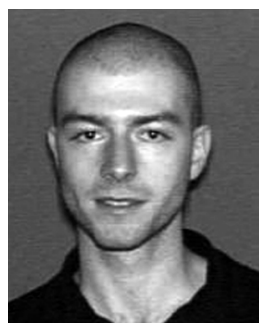

Bart De Wachter is with Interuniversity MicroElectronics Center (IMEC), Leuven, Belgium, Process Technology unit as a Researcher.

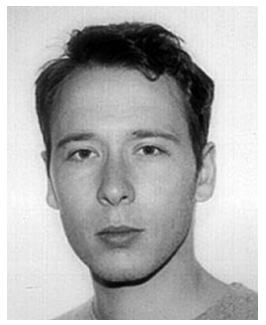

Morin Dehan received the B.S. and Ph.D. degrees in applied sciences from the Université Catholique de Louvain, Louvain-la-Neuve, Belgium, in 1999 and 2003, respectively.

Since 2004, he has been with Interuniversity MicroElectronics Center (IMEC), Leuven, Belgium. He worked on modeling, and on the optimization of passive components, fabricated in a CMOS process. Recently, he has been working on setting up PDKs for the different imec technologies, for both 2-D and 3-D designs.

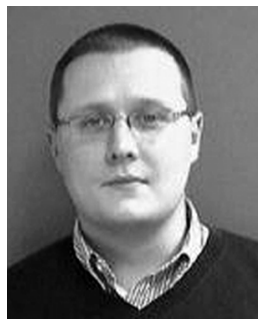

Marc Nelis was born in Liège, Belgium. He received the M.Sc. degree from the Université de Liege in 2005.

From 2006 to 2007 he worked at NXP Semiconductors as Test \& Product Engineer in the Power Management Unit. End of 2007 he joined Interuniversity MicroElectronics Center (IMEC), Leuven, Belgium, (PQI) as Test Chip Engineer and is active in process test structures layout for new technologies and data preparation for reticle.

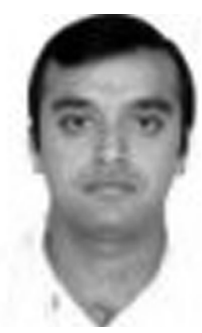

Rahul Agarwal is currently Senior Research Engineer at the Institute of Microelectronics (IME), Singapore.

From 2008 to 2010 he worked as researcher in 3-D packaging at Interuniversity MicroElectronics Center (IMEC), Leuven, Belgium, from 2001 to 2007 he was Research Assistant at University of South Florida.

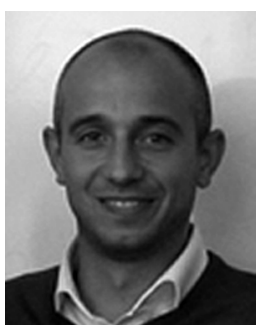

Antonio Pullini received the M.S. degree in electrical engineering from Bologna University, Italy.

$\mathrm{He}$ is a Senior Engineer at iNoCs S.à.r.l., Lausanne, Switzerland. His research interests include low-power digital design and networks on chip.

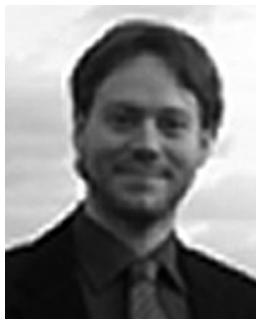

Federico Angiolini received the M.S. and Ph.D. degrees in electrical engineering from Bologna University, Italy, on the subject of Networks-on-Chips.

He is the VP of Engineering at iNoCs S.à.r.l., Lausanne, Switzerland. His background is on architectural design and simulation environments. 


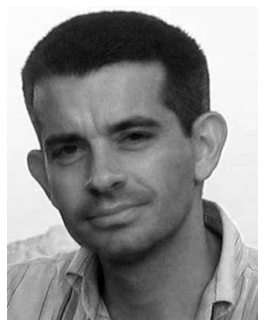

Luca Benini (F'07) is a Full Professor in the Department of Electronics and Computer Science, University of Bologna.

$\mathrm{He}$ also holds a Visiting Professor position at Ecole Federale Polytechnique de Lausanne. His research interests are in all the aspects of the computer-aided design of digital circuits, with special emphasis on low-power applications, and in the design of portable systems. On these topics, he published more than 500 papers.

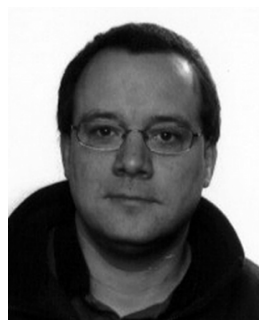

Wim Dehaene (SM'99) received the M.Sc. degree in electrical and mechanical engineering in 1991 and the Ph.D. degree in 1996 from the Katholieke Universiteit Leuven, Belgium. His thesis is entitled "CMOS integrated circuits for analog signal processing in hard disk systems."

After receiving the M.S. degree, he was a Research Assistant at the ESAT-MICAS Laboratory of the Katholieke Universiteit Leuven. His research involved the design of novel CMOS building blocks for hard disk systems. The research was first sponsored by the IWONL (Belgian Institute for Science and Research in Industry and agriculture) and later by the IWT (the Flemish institute for Scientific Research in the Industry). In November 1996 he joined Alcatel Microelectronics, Belgium. There he was a senior project leader for the feasibility, design and development of mixed mode Systems on Chip. The application domains were telephony, xDSL and high speed wireless LAN. In July 2002 he joined the staff of the ESATMICAS laboratory of the Katholieke Universiteit Leuven where he is now a Full Professor. His research domain is circuit level design of digital circuits. The current focus is on ultra low power signal processing and memories in advanced CMOS technologies. Part of this research is performed in cooperation with IMEC, Belgium where he is also a part time principal scientist. He is teaching several classes on electrical engineering and digital circuit and system design.

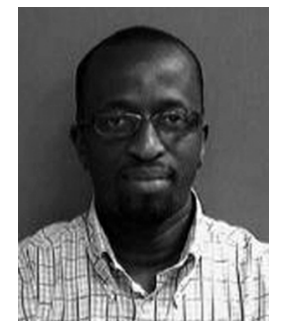

Youssef Travaly received the Ph.D. degree in materials science combined from Louvan-La-Neuve University, Belgium in 1997, and the MBA from Vlerick School of Management, Belgium, in 2009.

He has more than 16 years of cumulative work in semiconductor technology. He has authored and co-authored more than 100 journals and conference papers in the fields on interconnects. He is currently Program Manager of the 3-D integration program at Interuniversity Microelectronics Research center (IMEC), Leuven, Belgium.

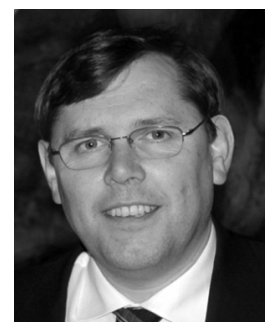

Eric Beyne received the degree in electrical engineering in 1983 and the Ph.D. in applied sciences in 1990, both from the Katholieke Universiteit Leuven, Belgium.

Since 1986 he has been with Interuniversity Microelectronics Research center (IMEC), Leuven, Belgium. Currently, he is Scientific Director of the advanced packaging and interconnect research, APIC, at IMEC. The APIC group performs R\&D in the field of. high-density interconnection and packaging techniques focused on "system-in-a-package" integration, 3-D-interconnections, wafer level packaging, rf front-end design and technology using integrated passives and rf-MEMS as well as research on packaging reliability including thermal and thermo-mechanical characterization.

Dr. Beyne is elected member of the board of governors of the IEEE CPMT society, president of the IMAPS-Benelux committee and member of the IMAPSEurope Liaison committee.

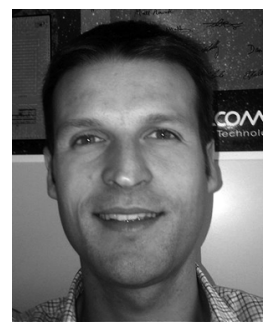

Paul Marchal received the engineering degree and $\mathrm{Ph} . \mathrm{D}$. degree in electrical engineering from the Katholieke Universiteit Leuven, Belgium in 1999 and 2005 , respectively.

He currently holds a position as Principal Scientist at Interuniversity Microelectronics Research center (IMEC), Leuven, Belgium. His team has as objective to set up a reference design flow for 3-D integration technology in collaboration with all partners of the supply chain (EDA vendors, foundries, packaging houses and design houses). 